\title{
Asymmetric shocks in $\chi$ Cygni observed with linear spectropolarimetry ${ }^{\star}$
}

\author{
A. López Ariste ${ }^{1}$, B. Tessore ${ }^{2}$, E. S. Carlín ${ }^{3,5,6}$, Ph. Mathias $^{1}$, A. Lèbre ${ }^{4}$, J. Morin $^{4}$, P. Petit ${ }^{1}$, \\ M. Aurière ${ }^{1}$, D. Gillet ${ }^{7}$, and F. Herpin ${ }^{8}$
}

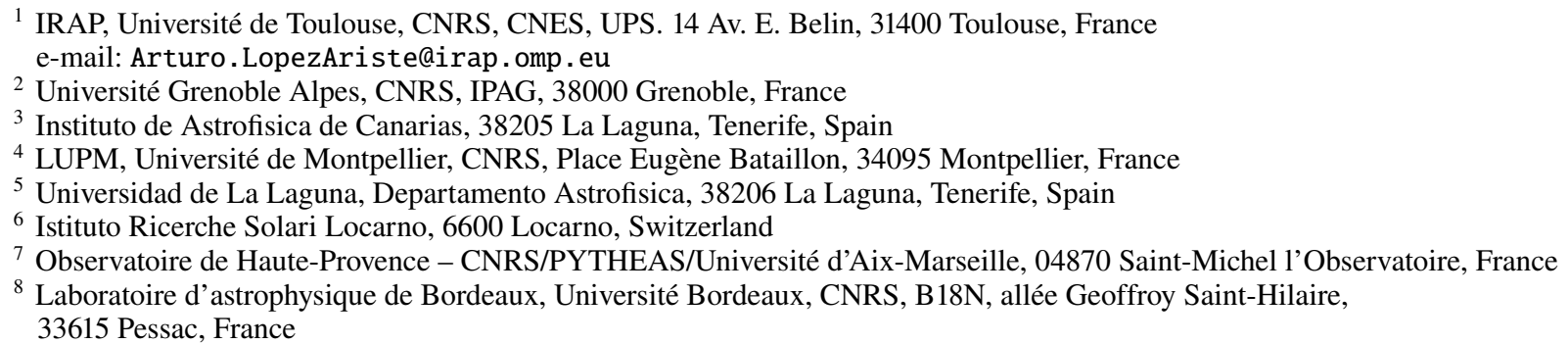

Received 27 June 2019 / Accepted 5 September 2019

\begin{abstract}
Aims. We derive information about the dynamics of the stellar photosphere, including pulsation, from a coherent interpretation of the linear polarisation detected in the spectral lines of the Mira star $\chi$ Cyg.

Methods. From spectropolarimetric observations of $\chi$ Cyg, we performed a careful analysis of the polarisation signals observed in atomic and molecular lines, both in absorption and emission, using radiative transfer in the context of polarisation produced through two mechanisms: intrinsic polarisation and continuum depolarisation. We also explain the observed line doubling phenomenon in terms of an expanding shell in spherical geometry, which allows us to pinpoint the coordinates over the stellar disc with enhanced polarisation.

Results. We find that the polarised spectrum of $\chi$ Cyg is dominated by intrinsic polarisation and has a negligible continuum depolarisation. The observed polarised signals can only be explained by assuming that this polarisation is locally enhanced by velocity fields. During the pulsation, radial velocities are not homogeneous over the disc. We map these regions of enhanced velocities.

Conclusions. We set an algorithm to distinguish the origin of this polarisation in any stellar spectra of linear polarisation and to find a way to increase the signal by coherently adding many lines with an appropriated weight. Applied to the Mira star $\chi$ Cyg, we reached the unexpected result that during the pulsation, velocities are radial but not homogeneous over the disc. The reason for these local velocity enhancements are probably related to the interplay between the atmospheric pulsation dynamics and the underlying stellar convection.
\end{abstract}

Key words. stars: variables: general - stars: imaging - techniques: polarimetric

\section{Introduction}

The recent discovery of strong linear polarisation signals in atomic lines of cool and evolved stars has spurred an interest in interpreting these signatures and exploiting them to learn about photospheric conditions of these stars using new diagnostics. Betelgeuse provided the first case of study of this kind. Aurière et al. (2016) described those signals and interpreted them as due to the depolarisation of the continuum. The fact that some signal remains after integration over the stellar disc implies that photospheric (brightness) inhomogeneities are present. From this interpretation, López Ariste et al. (2018) were able to map this brightness distribution over the stellar disc and to relate this distribution to the supergranulation of Betelgeuse. The reconstructed images obtained with this new technique applied to spectropolarimetric observations were corroborated by comparison with interferometric observations. Over four years, the

\footnotetext{
${ }^{\star}$ Based on observations obtained at the Télescope Bernard Lyot (TBL) at Observatoire du Pic du Midi, CNRS/INSU and Université de Toulouse, France.
}

granulation of Betelgeuse has been imaged, its spatial and temporal scales measured, and the velocities associated with this plasma estimated, altogether confirming that convection and granulation are at the origin of these signals (Mathias et al. 2018). The technique has since been extended to two more red supergiants: CE Tau and $\mu$ Cep (Tessore et al., in prep.).

In an attempt to generalise this successful result, other types of cool and evolved stars have been considered for similar linear polarisation signals associated with spectral lines. One of these stars is the Mira star $\chi$ Cyg. Clear linear polarisation signals have indeed already been detected in the Balmer lines of Mira stars (e.g. in the prototypical Mira star $o$ Ceti; see Fabas et al. 2011), but, more important for the present work, polarisation signals were also detected in many other atomic and molecular lines over the spectrum of $\chi$ Cyg (Lèbre et al. 2015).

The interpretation of these linear polarimetric signals associated with spectral lines is the purpose of this work. In Sect. 2, we present the Mira star $\chi$ Cyg and all the observational material involved in this work. In Sect. 3, we investigate the origin of the linear polarisation associated with spectral lines. Starting from the signatures within the line profiles that suggest 
intrinsic polarisation, we developed several tests to confirm this hypothesis. This kind of approach, with tests confirming initial hypothesis, is also used to interpret the nature of the surface inhomogeneities, which is necessary for the polarisation signal to survive its integration over the stellar disc, pointing in particular to local velocity increases. Our main result is that the pulsation of the Mira star $\chi$ Cyg, while being radial in direction, presents different velocities at different places over the disc. In Sect. 5, we exploit the data from the spectropolarimetric survey we performed on $\chi$ Cyg in 2015 and 2016 to map carefully the places at which these velocity enhancements appear. We speculate, in Sect. 6, that these velocity enhancements may be the result of the interaction of the pulsation mechanism with either the general convective patterns or the returning material from previous cycles.

\section{Spectropolarimetry of the Mira star $\chi$ Cyg}

\subsection{Mira star $\chi$ Cyg}

Mira variables are low- to intermediate-mass asymptotic giant branch (AGB) stars that pulsate with a period of about one year. $\chi$ Cyg is an S-type Mira star of spectral type S6-9/1-2e that has a pulsation period of about $408 \mathrm{~d}$. High-resolution spectroscopic studies of Mira stars (Gillet et al. 1983; Alvarez et al. 2000, 2001) have reported strong emissions of the hydrogen lines lasting up to $80 \%$ of the luminosity period. Those works have established that radiative and hypersonic shock waves, which are triggered by the pulsation mechanism, periodically propagate throughout the stellar atmosphere, generating emission lines formation process and favouring the doubling of metallic lines. Well beyond the region the atmospheric regions probed with our data, the stellar neighbourhood of Mira stars has also been thoroughly studied through observations of $\mathrm{SiO}$ masers or $\mathrm{CN}$ emissions (e.g. Herpin et al. 2006; Duthu et al. 2017); these studies allowed for estimations of the magnetic field strength in the circumstellar envelope, i.e. up to 8.8 gauss in $\chi \mathrm{Cyg}$ at a few stellar radii. Vlemmings et al. (2017) observed magnetically aligned dust and $\mathrm{SiO}$ maser polarisation in the envelope of the red supergiant VY Canis Majoris. All these observations are consistent with a toroidal field configuration in these objects.

From full Stokes spectropolarimetric observations, Fabas et al. (2011) characterised the shock wave propagation throughout the stellar atmosphere of the prototypical oxygen-rich Mira star, $o$ Ceti. These authors reported signatures in Stokes $Q$ and $U$ but also in Stokes $V$ parameters, tracing linear and circular polarisation, respectively, associated with the strong Balmer hydrogen emissions known to be formed in the radiative wake of the shock wave (Fadeyev \& Gillet 2004). The origin of these spectropolarimetric signatures reveals a global asymmetry (at least partly photospheric) perhaps due to the passage of the front of the shock throughout photospheric giant convective cells. A few years later, Lèbre et al. (2014) reported the first detection of a faint magnetic field at the surface of the S-type Mira $\chi$ Cyg, that is still to date the unique detection of a surface magnetic field for Mira stars. Lèbre et al. (2015) also reported for $\chi$ Cyg strong signatures in Stokes $Q$ and $U$ profiles associated with metallic lines. These features are strong, since they are detected from single observing sequences and they are also variable along the pulsating phase. The positions of these striking Stokes $Q$ and $U$ profiles was found to be well connected to the shock front position. Moreover, Lèbre et al. (2015) also reported, from the Stokes $U$ and Stokes $Q$ spectra of $\chi$ Cyg, clear signatures associated with individual lines (e.g. SrI at $460.7 \mathrm{~nm}$,
$\mathrm{Na}$ D2 at $588.9 \mathrm{~nm})$. In the solar case, these peculiar lines are known to be easily polarisable in the presence of asymmetries at the photospheric level; an extensive bibliography of the solar case as well as a theoretical introduction to the problem of polarized line formation in stellar atmospheres can be found in Landi Degl'Innocenti \& Landolfi (2004).

\subsection{Spectropolarimetric observations of $\chi$ Cyg}

Table 1 shows the series of observations of $\chi$ Cyg at our disposal for the present work. Except for the first observation (September 4, 2007) performed during a previous observing campaign, we obtained all the 2015-2016 observations in the same large programme conducted with the Narval spectropolarimetric instrument mounted at the Telescope Bernard Lyot (TBL; Pic du Midi, France).

Figure 1 shows the time series of the visual magnitude of $\chi$ Cyg as measured by the American Association of Variable Star Observers (AAVSO). The vertical dashed lines indicate our available 2015-2016 observations, considering that some observations collected at close dates (or consecutive nights) have been combined into one observational phase (cf. Table 1). All these observations appear to have been collected around a maximum light ( $\phi$ from 0.8 to 1.3 ), when the atmospheric shock wave is known to have constantly an upward motion while propagating throughout the stellar atmosphere (Gillet et al. 1985). The Stokes $U$ and Stokes $Q$ observations of September 4, 2007 (also collected with Narval at TBL) show clear signatures in individual lines. Since these were also the first data to be scrutinised, these 2007 observations are further used to illustrate the origin of the linear polarisation associated with atomic and molecular lines (see Sect. 3). The 2015-2016 observations, resulting from a regular spectropolarimetric monitoring of $\chi$ Cyg (on a monthly basis when the star was observable from Pic du Midi), are used in Sect. 4 to map photospheric conditions.

\section{Origin of the linear polarisation associated with the spectral lines of $\chi$ Cyg}

Polarisation signals in stellar atomic lines are weak, often below $0.1 \%$ of the continuum intensity. It is customary in stellar spectropolarimetry to add the signals over many lines throughout the spectrum with the goal of increasing the signal-to-noise ratio (S/N). Such algorithms, for example least squares deconvolution (LSD; Donati et al. 1997; Kochukhov et al. 2010), single line addition (SLA; Paletou 2012) and others, depend on the selfsimilarity assumption; according to this assumption all lines to be co-added are formed at similar heights and carry a signal with a similar spectral shape up to a scale factor. This has been traditionally the case of circular polarisation that is related to the Zeeman effect and used to detect surface magnetic fields.

The situation faced in this work with $\chi$ Cyg is that the S/Ns are barely sufficient to be reliable only through the LSD technique. But trusting LSD implies that the signatures in individual lines are similar, although the $\mathrm{S} / \mathrm{N}$ in those lines is in general too low to check this assumption. In order to justify the use of LSD to study linear polarisation of $\chi \mathrm{Cyg}$, we started with an in-depth analysis of the signals detected in key individual lines.

Among the observations of $\chi$ Cyg (presented in Table 1), several observations stand out because of the large amplitude of the linear polarisation signals over most of the visible spectrum. The largest amplitudes seen (September 4, 2007 or August 8 and $16,2007)$ coincide with the dates around the maximum light (phases 0.91, 0.94, and 0.95). We picked one of such singular 
Table 1. Linear polarisation observations of $\chi$ Cyg (Stokes $U$ and/or Stokes $Q$ ) from September 2007 to December 2016.

\begin{tabular}{llccccc}
\hline \hline Stokes & $\begin{array}{l}\text { Obs. date } \\
(\mathrm{dd} / \mathrm{mm} / \mathrm{yyyy})\end{array}$ & $\begin{array}{c}\text { Julian date }(\mathrm{JD}) \\
(+2450000)\end{array}$ & $\begin{array}{c}\text { Phase } \\
(\phi)\end{array}$ & $\begin{array}{c}\text { Exposure } \\
\text { (in s) }\end{array}$ & $\begin{array}{c}N \\
(Q \text { and } U)\end{array}$ & $\begin{array}{c}S / N \\
\left(/ 2.6 \mathrm{~km} \mathrm{~s}^{-1}\right)\end{array}$ \\
\hline$Q$ and $U$ & $04 / 09 / 2007$ & 4347.86 & 0.94 & 400 & $1 \& 1$ & 1527 \\
$Q$ and $U$ & $28 / 05 / 2015$ & 7171.11 & 0.84 & 400 & $2 \& 2$ & 1464 \\
$Q$ and $U$ & $01 / 06 / 2015$ & 7175.12 & 0.85 & 400 & $2 \& 2$ & 1488 \\
$Q$ and $U$ & $26 / 06 / 2015^{(a)}$ & 7200.05 & 0.91 & 400 & $1 \& 1$ & 1369 \\
$Q$ and $U$ & $28 / 06 / 2015^{(a)}$ & 7202.02 & 0.92 & 400 & $1 \& 1$ & 1479 \\
$Q$ and $U$ & $19 / 07 / 2015$ & 7222.98 & 0.97 & 400 & $3 \& 4$ & 1391 \\
$Q$ and $U$ & $10 / 08 / 2015$ & 7245.01 & 0.02 & 400 & $4 \& 4$ & 990 \\
$Q$ and $U$ & $20 / 08 / 2015$ & 7254.91 & 0.05 & 400 & $4 \& 4$ & 1270 \\
$Q$ and $U$ & $10 / 09 / 2015^{(a)}$ & 7275.96 & 0.10 & 400 & $2 \& 2$ & 1470 \\
$Q$ and $U$ & $11 / 09 / 2015^{(a)}$ & 7276.82 & 0.10 & 400 & $2 \& 2$ & 1260 \\
$Q$ and $U$ & $09 / 10 / 2015$ & 7304.93 & 0.17 & 400 & $2 \& 2$ & 1568 \\
$Q$ and $U$ & $16 / 10 / 2015$ & 7311.80 & 0.19 & 400 & $2 \& 2$ & 904 \\
$Q$ and $U$ & $15 / 11 / 2015^{(a)}$ & 7341.86 & 0.26 & 400 & $2 \& 2$ & 882 \\
$Q$ and $U$ & $16 / 11 / 2015^{(a)}$ & 7342.74 & 0.26 & 400 & $2 \& 2$ & 1048 \\
$Q$ and $U$ & $07 / 08 / 2016^{(a)}$ & 7607.88 & 0.91 & 400 & $4 \& 4$ & 1349 \\
$Q$ and $U$ & $08 / 08 / 2016^{(a)}$ & 7608.89 & 0.92 & 400 & $4 \& 4$ & 1347 \\
$Q$ and $U$ & $24 / 08 / 2016$ & 7625.00 & 0.95 & 400 & $4 \& 4$ & 1556 \\
$Q$ and $U$ & $24 / 09 / 2016$ & 7655.90 & 0.03 & 400 & $3 \& 3$ & 1096 \\
$Q$ and $U$ & $21 / 10 / 2016$ & 7682.83 & 0.09 & 400 & $3 \& 3$ & 1183 \\
$\mathrm{Q}$ & $26 / 10 / 2016^{(b)}$ & 7687.86 & 0.11 & 400 & 3 & 909 \\
U & $27 / 10 / 2016^{(b)}$ & 7687.86 & 0.11 & 400 & 3 & 682 \\
$Q$ and $U$ & $07 / 12 / 2016$ & 7729.73 & 0.21 & 400 & $4 \& 4$ & 1548 \\
$Q$ and $U$ & $20 / 12 / 2016$ & 7742.75 & 0.24 & 400 & $4 \& 4$ & 1224 \\
\hline
\end{tabular}

Notes. For each observation, the first column gives the observed Stokes parameter(s). The second and third columns give the date of observation in the Gregorian and Julian calendars, respectively. The fourth column gives the phase of the star $(\phi)$, considering an ephemeris giving $\phi=0$ at maximum light for JD $=2457234.4$ (30 July 2015) and a period of 408.7 days. All the observations have been replaced on a single pseudo-cycle (from $\phi=0.00$ to $\phi=1.00$ ). The last columns give the exposure time (in seconds), number of spectra for each parameter and the maximum $\mathrm{S} / \mathrm{N}$ in each spectrum, respectively. ${ }^{(a)}$ Observations that have been pushed together. ${ }^{(b)}$ One Stokes parameter is missing for this date, therefore this parameter has not been considered in our study.

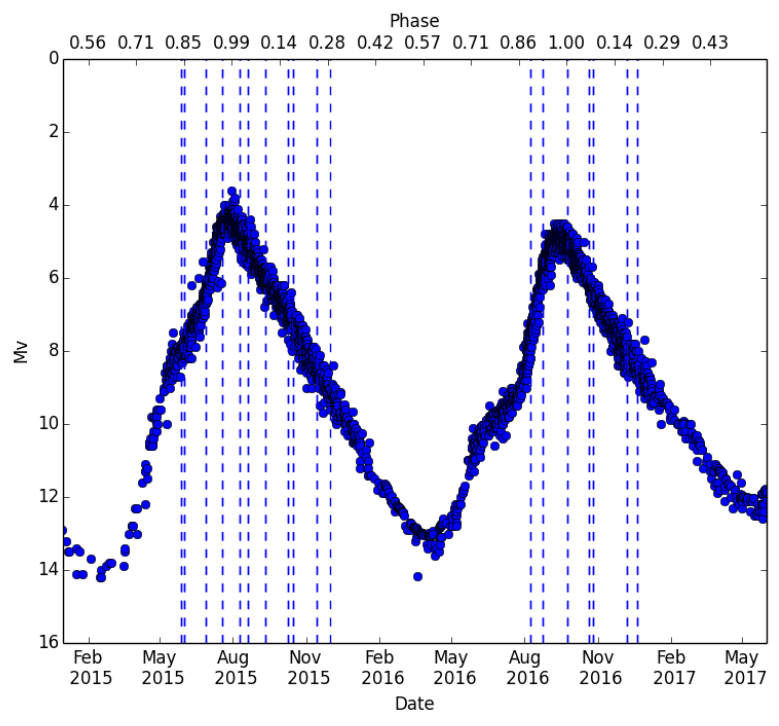

Fig. 1. Visual magnitude of $\chi$ Cyg as measured by AAVSO. The vertical dashed lines indicate the dates for which spectropolarimetric data are available.

observations, that of September 4, 2007, close to the occurrence of a maximum light $(\phi=0.94)$. According to the shock propagation scenario from Gillet et al. (1985) this observation corresponds to the following pulsation state for a Mira star: a strong shock wave has already emerged from the photosphere (just before the maximum light, at around $\phi=0.8$ ) and is propagating throughout the stellar atmosphere, until its final fading around $\phi=1.4$. At this stage the observation of September 4, 2007 produced strong signals, sufficient to examine individual lines and reveal the physical mechanisms at work in the production of linearly polarised light.

\subsection{Radiative transfer equation}

Roughly speaking, linear polarisation in atomic lines of a stellar spectrum can have two origins: Zeeman effect or scattering. For $\chi$ Cyg, Lèbre et al. (2014) detected a very weak circular polarisation signature around its maximum light of March 2012 , revealing the presence of a faint surface magnetic field (2-3 G). However, its effect on the linear polarisation is expected to be one order of magnitude smaller. Therefore, in the case of $\chi$ Cyg, a Zeeman contamination of the linear polarisation can be excluded. And thus we retain in the following only scattering processes.

In this context, within the stellar atmosphere, an atom has three different ways of creating a spectral polarisation signature: continuum depolarisation through lines, intrinsic line polarisation, and finally Rayleigh and/or Thomson scattering. We followed Landi Degl'Innocenti \& Landolfi (2004) to write, in the absence of magnetic fields, a radiative transfer equation for 
the Stokes parameter $Q$, which is defined as positive when the polarisation plane is perpendicular to the scattering plane ${ }^{1}$ of a particular point in the stellar atmosphere. Taking advantage that this polarisation is always small, this equation is written as

$$
\begin{aligned}
& \frac{\mathrm{d}}{\mathrm{d} s} Q(v, \boldsymbol{\Omega})=-\left[k_{v}^{c}+k_{L}^{A} \phi\left(v_{0}-v\right)\right] Q(v, \boldsymbol{\Omega}) \\
& \quad+\frac{3}{2 \sqrt{2}} k_{L}^{A} \sin ^{2} \theta\left[w_{J_{u} J_{l}}^{(2)} \sigma_{0}^{2}\left(J_{u}\right) S_{L}-w_{J_{l} J_{u}}^{(2)} \sigma_{0}^{2}\left(J_{l}\right) I(v, \boldsymbol{\Omega})\right] \phi\left(v_{0}-v\right) \\
& \quad+\frac{3}{2 \sqrt{2}} k_{v}^{c} \sin ^{2} \theta \sum_{i=1}^{3} \beta_{i} \int \mathrm{d}^{3} \boldsymbol{v}_{i} f\left(\boldsymbol{v}_{i}\right)\left[J_{0}^{2}\left(v-v \frac{\boldsymbol{v}_{i} \cdot \boldsymbol{\Omega}}{c}\right)\right]_{v_{i}}
\end{aligned}
$$

where the three considered processes quoted above correspond to each line on the right-hand side, respectively.

The equation describes the evolution of the Stokes $Q$ parameter along a path $s$ as a function of frequency $v$ and in the direction $\boldsymbol{\Omega}$. An atomic line is assumed to be at $v_{0}$ in the proximity of the frequency $v$ with a characteristic line profile described by $\phi\left(v_{0}-v\right)$. In spite of being a first order approximation, this equation is very complex at first sight. It unveils the richness of the scattering polarisation spectra of a star, even in the absence of magnetic fields. It is this richness that led Stenflo \& Keller (1997) to coin the name second solar spectrum to refer to this scattering polarisation spectrum in the case of the Sun. Any hope in using LSD techniques requires the identification of a large enough number of atomic lines that are sensitive to just one of those three processes made explicit in the right-hand side of this equation.

\subsubsection{Rayleigh and/or Thompson scattering}

In the absence of lines sensitive to the first two mechanisms in Eq. (1), the only non-zero term in the equation would be the last term, which describes Rayleigh and/or Thompson scattering of photons over electrons $(i=1), \mathrm{H}$ atoms $(i=2)$, and He atoms $(i=3)$. Scattering over heavier atoms can be safely neglected. Rayleigh scattering linearly polarises light proportionally to $\sin ^{2} \theta$, the scattering angle. The amount of light is obviously proportional to the absorption coefficient of the continuum at that frequency $k_{v}^{c}$, but also to the fractional contribution $\beta_{i}$ of electrons, $\mathrm{H}$, and He atoms to that opacity. For a scatterer to emit net polarisation, it should be illuminated by an anisotropic radiation field described by the non-zero, secondrank spherical tensor of the radiation field $J_{0}^{2}$, which in the first approximation can be written as $3 K-J$ where $K$ and $J$ are the second and zeroth order momenta of the angular distribution of the specific intensity; this radiation field depends on frequency in the rest frame of the scatterer. An integral over the velocity distribution $f\left(\boldsymbol{v}_{i}\right)$ is necessary to convert this dependence to the observer's frame. If the continuum forms over a large enough atmospheric layer for anisotropy to be non-negligible, we expect the continuum to be polarised. In the Sun this continuum polarisation amounts to $0.1 \%$ (Leroy 1972; Stenflo 2005). In cool stars like Betelgeuse ( $\alpha$ Ori) the polarisation grows to $1 \%$ of the continuum intensity (Clarke \& Schwarz 1984; Doherty 1986). In the Mira star $\chi$ Cyg, Boyle et al. (1986) reported (from polarimetric observations collected around a maximum light) that the polarisation of the continuum varies from $1 \%$ in the blue part down to $0.25 \%$ in the red part of the spectrum. They also reported striking enhancements in the linear polarisation level associated with

1 This plane is defined by the scattering point, the centre of the star, and the observer.
Balmer emission lines $(+\sim 0.5 \%)$, to molecular bands $(+\sim 0.5 \%)$, and to the $\mathrm{Ca}$ I line at $422.6 \mathrm{~nm}(+\sim 2 \%)$ pointing to a polarisation likely arising from the stellar atmosphere in the region of formation of the spectral lines.

\subsubsection{Continuum depolarisation}

A spectral line that forms above a continuum polarised by Rayleigh scattering absorbs linearly polarised photons and reemits a priori unpolarised photons. Spectral lines therefore tend to depolarise the continuum. This process is described by the first term of Eq. (1), where this depolarisation of the incoming polarisation $Q(v, \boldsymbol{\Omega})$ is described as proportional to the combined absorption coefficients of the continuum and the line, $k_{v}^{c}+k_{L}^{A}$, times the spectral profile of the line. We depend on the details of the stellar atmosphere with respect to the formation regions of the continuum and every individual lines to estimate the impact of this depolarisation mechanism on the second stellar spectrum. In the case of the Sun, well over $90 \%$ of the spectral lines depolarise the continuum. In particular, with few exceptions, all the numerous $\mathrm{Fe} I$ lines depolarise the continuum. Aurière et al. (2016) found that, in the case of Betelgeuse, all atomic lines depolarised the continuum and since the spectral shape of a line $\phi\left(v_{0}-v\right)$ can be considered similar enough to that of any other line, this allowed those authors to use LSD on the linearly polarised spectrum of Betelgeuse and to map the presence of bright spots on its photosphere.

\subsubsection{Intrinsic line polarisation}

Competing with depolarisation, we find the second and most complex term of Eq. (1). This term describes what we refer to as the intrinsic polarisation of a line $e^{2}$. Anisotropic illumination can introduce both population imbalances or coherences among otherwise degenerated atomic sub-levels. These coherences are quantum in nature and in the present case reduce to the $\sigma_{0}^{2}(J)$ spherical tensor of the atomic density matrix corresponding to the level with total angular momentum $J$, an imbalance of populations between sub-levels that we call atomic alignment. Atomic alignment in the upper level results in polarisation in the emitted light, and this is why we multiply it by the source function $S_{L}$. Atomic alignment in the lower level can be seen as absorbed incoming photons, and this is why we multiply it by the incoming intensity $I(v, \boldsymbol{\Omega})$. Not all transitions between atomic levels are equally efficient in producing polarisation in the presence of atomic alignment: the $w_{J_{u} J_{l}}^{(2)}$ quantum coefficient expresses this efficiency as a function of the upper and lower total angular momenta. Its explicit definition can be found for instance in Landi Degl'Innocenti \& Landolfi (2004). Particular values of the upper and lower angular momenta cancel this coefficient. For example transitions from $\left(J_{l}=\right) \frac{1}{2}$ to $\left(J_{u}=\right) \frac{1}{2}$, or from 1 to 0 , or from $\frac{3}{2}$ to $\frac{1}{2}$, have all $w_{J_{u} J_{l}}^{(2)}=0$. All those atomic lines in the stellar spectra with those values of the total angular momentum for their lower and upper levels are unable to produce intrinsic polarisation in consequence, any polarisation signal in such selected lines may only be attributed to depolarisation. In the presence of

\footnotetext{
2 In contrast to previous uses by other authors of the adjective intrinsic and at the risk of some confusion, we call intrinsic any signal with origin in this term which does not explicitly depend on the incoming light or its polarisation as the two other terms. Evidently, the atomic polarisation appearing in this second term may have its origin in anisotropic radiation, but as explained, there is a clear difference in the type of signals expected from one and the other terms in Eq. (1) hence justifying our use of intrinsic.
} 

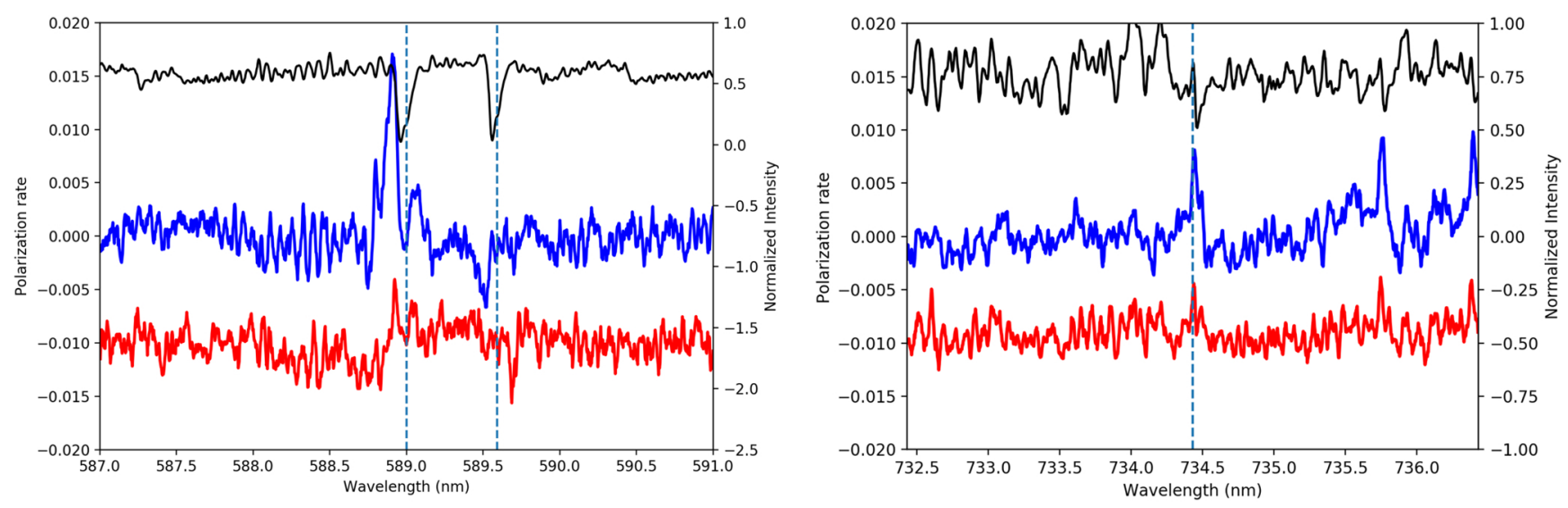

Fig. 2. Spectra of $\chi$ Cyg on September 4, 2007. Left: around the region of the Na D doublet; right: around a Ti I line in the near-infrared spectrum. The thin black line shows the intensity (ordinate axis on the right) in normalised units, whereas the thick coloured lines (blue for $U$, red for $Q$ ) show the linear polarisation spectra. The rest wavelength of both $\mathrm{Na} D_{1}$ and $D_{2}$ in the left plot and the Ti I line in the right plot are represented with a vertical dashed line.

noise, these conclusions can be extended to other cases with low absolute values of $w_{J_{u} J_{l}}^{(2)}$.

This provides one possible way to distinguish the origin of observed linear polarisation in a stellar spectrum. Following Aurière et al. (2016) we consider the $\mathrm{Na} D$ doublet. Neglecting hyperfine structure, the $D_{1}$ line arises from a $\frac{1}{2}-\frac{1}{2}$ transition with $w_{\frac{1}{2}, \frac{1}{2}}^{(2)}=0$. This means that the $D_{1}$ line cannot produce intrinsic polarisation. We note that the hyperfine structure modifies the past assertion: among the several hyperfine transitions that form the $D_{1}$ line, some can indeed carry intrinsic polarisation. This can be seen in the second solar spectrum, where the $D_{1}$ line indeed shows a signal (Casini et al. 2002; Stenflo \& Keller 1997; Trujillo Bueno et al. 2002). But this is a signal at least one order of magnitude weaker than that present in $D_{2}$. Indeed, $D_{2}$ has $w_{\frac{3}{2}, \frac{1}{2}}^{(2)}=0.6$ and it is prone to show strong intrinsic signals. Both $D_{1}$ and $D_{2}$ components are, on the other hand, equally efficient in depolarising the continuum and have almost identical conditions of formation and frequency.

By looking into the second spectrum of $\mathrm{Na} D$ lines, we can therefore identify two extremes: if $D_{1}$ shows a polarisation signal with a similar amplitude as that of $D_{2}$, then the lines are depolarising the continuum. On the other hand, if the $D_{1}$ linear polarisation is much smaller than that of $D_{2}$, then the signals are dominated by intrinsic polarisation.

The first scenario was found by Aurière et al. (2016) on Betelgeuse: both $D_{1}$ and $D_{2}$ components showed similar amplitudes, and the interpretation was that the atmospheric structure in that star leads to a depolarisation of the continuum (first term of Eq. (1)). Indeed, if $D_{2}$, a line so prone to show strong intrinsic signals is depolarising continuum, we can safely assume that so do all the lines in the spectrum. The Sun itself shows a mixed scenario. While most of the lines just depolarise the continuum, a few lines form in conditions adequate to show intrinsic polarisation, $D_{2}$ prime among them.

\subsection{Dominating process for $\chi$ Cyg}

We now consider the $\mathrm{Na} D$ doublet in the spectrum of $\chi \mathrm{Cyg}$ obtained on September 4, 2007 (Fig. 2). We note that even considering a night with one of the largest linear polarimetric signals, the amplitude of the polarisation signature is weak. This is partly because of the low $\mathrm{S} / \mathrm{N}$ in individual lines, but also the ubiquitous presence of molecular lines (see below). In spite of those problems, we can identify a double peak around $D_{2}$, positive in both $Q$ and $U$, whereas nothing is present in the same wavelength range around $D_{1}$. Small peaks with opposite amplitudes are seen right beyond the red wing of $D_{1}$ in $Q$ and the blue wing in $U$. The coherence of polarisation signs in all absorption lines that we are going to uncover in the text below, and the absence of any spectral feature at this wavelength, led us to ignore these signals; the second solar spectrum shows a weak signal in $D_{1}$ that is one order of magnitude smaller than the signal in $D_{2}$, but it remains difficult to relate this signal to the peaks seen in our data. With this caveat in mind, we find in this absence of signal in $D_{1}$ a first indication that intrinsic polarisation dominates the $\mathrm{Na} D$ lines for $\chi$ Cyg.

Also, Fig. 2 (right) shows, for the line Ti I at $734.4 \mathrm{~nm}$, signals in both $Q$ and $U$, signals that are of the same positive sign as those present in the $\mathrm{Na} D_{2}$ component. It is not the only signature with an identical sign in that region; around $736 \mathrm{~nm}$ another line (probably Ti I at $735.774 \mathrm{~nm}$ ) also shows this similar signature. We recall that while most lines present a single Gaussian-like polarisation profile, the $\mathrm{Na} D_{2}$ line presents a characteristic multi-peak profile as a result of its rich fine atomic structure. The comparison between this and lines such as the Ti I in this figure must be limited to the signs of the $Q$ and $U$ profiles.

A trend appears in which selected lines show a polarisation signature that happens to be positive in both $Q$ and $U$ for September 4, 2007 and, in the case of the $\mathrm{Na} D$ lines, this has to be interpreted as intrinsic polarisation. The set of lines showing analogous signatures is much larger, but it quickly gets lost in with the many molecular lines and bands in the spectrum of this cool star. This is the reason for having selected, for the plot, this otherwise unremarkable Ti I line around $736 \mathrm{~nm}$ : it is located in a region in which molecules do not appear to overwhelm the spectrum.

Molecular scattering polarisation is a much richer world than what we have explored until now (Asensio Ramos \& Trujillo Bueno 2005, 2006; Landi Degl'Innocenti \& Landolfi 2004). But in general, molecules present alignment and intrinsic line polarisation in an analogous manner to atoms (Landi Degl'Innocenti \& Landolfi 2004). We considered whether the $Q$ and $U$ signals common to both $\mathrm{Na} D_{2}$ and Ti I lines presented in Fig. 2 are also present in molecular lines. Figure 3 shows 

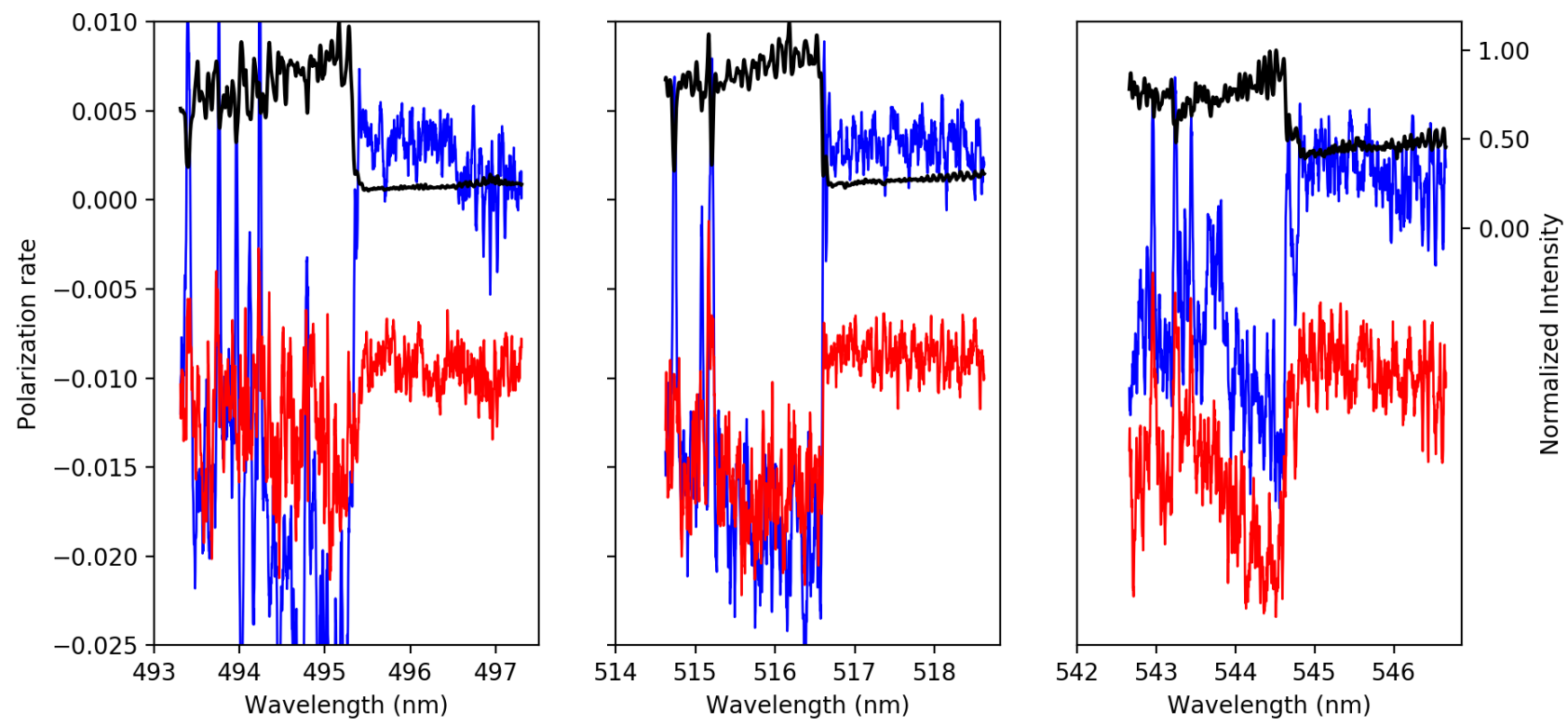

Fig. 3. Spectra of $\chi$ Cyg on September 4, 2007 in three spectral regions in which molecular band heads of TiO and VO are found. As in Fig. 2, the black thin line represents intensity (ordinates at right) and Stokes $Q$ and $U$ are shown in red and blue, respectively. The $Q$ profile has been shifted 0.01 units for clarity.

three different molecular band heads along the visible spectrum of $\chi$ Cyg. Molecular transitions cluster around the band head and concentrate the signal: the intensity goes down and linear polarisation also shows a drastic positive jump both in $Q$ and $U$.

Further examination of other molecular band heads and individual molecular lines confirm that a net linear polarisation signal positive both in $Q$ and $U$ is ubiquitous in molecular and in atomic lines. However, this signal is present in the $\mathrm{Na} \mathrm{D}_{2}$ line, but not in the $\mathrm{Na} D_{1}$ line. Hence, we make the hypothesis that all these atomic and molecular lines show intrinsic line polarisation through scattering in an aligned atom or molecule. Taking this hypothesis to its extreme, we cancel in Eq. (1) the continuum polarisation (third) and the line depolarisation (first) terms. Consequently, Eq. (1) becomes

$$
\begin{aligned}
Q= & \frac{3}{2 \sqrt{2}} k_{L}^{A} \sin ^{2} \theta\left[w_{J_{u} J_{l}}^{(2)} \sigma_{0}^{2}\left(J_{u}\right) S_{L}-w_{J_{l} J_{u}}^{(2)} \sigma_{0}^{2}\left(J_{l}\right) I(v, \mathbf{\Omega})\right] \\
& \times \phi\left(v_{0}-v\right) .
\end{aligned}
$$

In this equation all the spectral shape dependence around a given spectral line is in the generalised profile $\phi\left(v_{0}-v\right)$. This points towards the possibility of adding up lines through LSD (Donati et al. 1997) after appropriate re-scaling of the frequency. Of course, in this case, the weighting of the individual lines cannot be the Landé factor (the usual weighting in Zeeman Doppler Imaging; see Donati et al. 1997). Inspection of Eq. (2) allows further simplification by noticing that more often than not we can assume that the lower level of most transitions, because of its stability, is probably depolarised by collisions and therefore $\sigma_{0}^{2}\left(J_{l}\right)=0$. We also notice that in a two-level atom the alignment of the upper level is given by

$\sigma_{0}^{2}\left(J_{u}\right)=w_{J_{u} J_{l}}^{(2)} \frac{J_{0}^{2}}{J_{0}^{0}}$

and therefore the emitted polarisation can be approximated by

$Q=\frac{3}{2 \sqrt{2}} k_{L}^{A} \sin ^{2} \theta\left(w_{J_{u} J_{l}}^{(2)}\right)^{2} \frac{J_{0}^{2}}{J_{0}^{0}} \phi\left(v_{0}-v\right)$.
From this latter expression, we realise that the appropriate weighting factor for LSD is the coefficient $\left(w_{J_{u} J_{l}}^{(2)}\right)^{2}$, which depends exclusively on the atomic numbers of the upper and lower levels.

If the other two processes were also present, it could be noticed that independent of the sign of $w_{J_{u} J_{l}}^{(2)}$ (which can be either positive or negative depending on the quantum numbers of the involved levels), the emitted polarisation always has the same sign: perpendicular to the scattering plane. On the other hand, continuum polarisation is also perpendicular to this plane. Depolarisation cancels this signal, and it appears in our polarimeters as a signal of the opposite sign: parallel to the scattering plane. Thus, intrinsic line polarisation and depolarisation processeses have similar spectral shapes but opposite signs. If, contrary to our extreme assumption of all lines showing intrinsic line polarisation, a subset of lines shows a depolarisation signal (as it is the case in the second solar spectrum) the polarisation signal would cancel out when summing up lines, and the LSD profile would end up with an amplitude that is much smaller than that seen in individual lines such as those shown in Fig. 2. This provides us with an a posteriori test upon our hypothesis (Sect. 4).

\subsection{Polarisation signal and asymmetry}

Polarised stellar spectra are the result of the integration of the signal over the stellar disc. We found a source of local linear polarisation in the intrinsic polarisation of the lines formed by scattering. But since the scattering plane rotates with the position angle of the scattering point over the stellar disc, the signs of $Q$ and $U$ change around the stellar disc and, if the star is homogeneously polarising its spectrum, the result is a zero net signal. Since for $\chi$ Cyg a net polarisation is observed, we must conclude that the stellar disc is not centrally symmetric with respect to polarisation. We must conclude that there is one region of the stellar disc with a larger emission of polarised light.

There are two manners in which one particular region over the stellar disc can dominate the integral which computes the net polarisation over the star: either this region is brighter than the 
rest, or its light is more polarised. This second case of polarisation excess can also be split into two phenomena: either light is emitted from a higher region in the atmosphere, which translates into an increased anisotropy $J_{0}^{2}$ of the radiation field illuminating the atoms, or the emitting region is moving with respect to the photosphere, the presence of a velocity gradient amplifying the radiation anisotropy (de Kertanguy 1998). As a matter of fact, these three phenomena are not exclusive and we could, as an example, call for a convection cell bringing up hot plasma: hence brighter, in a strong up-flow (hence an anisotropy amplifying velocity gradient) that brings this plasma high in the atmosphere (hence increasing the anisotropy on its own).

When considering these non-exclusive alternatives we must keep in mind the pulsation of $\chi$ Cyg that may amplify any anisotropy present in the lower layers of the star. This leads us to consider that the origin of this anisotropy may be found in the non-homogeneity of the pulsation itself. The net observed polarisation would arise from the differential amplification of anisotropy by the higher velocity gradients of those regions expanding faster than all others, a hypothesis already hinted by Fabas et al. (2011) and proposed by Carlin et al. (2013) for the prototypical Mira star, $o$ Cet. In what follows we describe, step by step, how we conclude that this scenario is that at work in $\chi$ Cyg.

\subsection{Asymmetry from inhomogeneous velocity fields}

Carlin et al. (2012) studied the amplification of the anisotropy by velocity gradients along the vertical direction. A remarkable result of those studies is that emission and absorption lines produce opposite effects in the anisotropy: for otherwise identical lines, an amplification of the anisotropy happens if the line is in absorption but a reduction in anisotropy happens if it is in emission. Explicitly, a solution for the intensity around a spectral line of width $w$, with continuum at $I^{(0)}$, at a certain distance of disc centre $\mu$, and in the presence of a linear limb darkening with coefficient $u$ can be written as

$I(v, \mu)=I^{(0)}(1-u+u \mu)\left[1-a \mathrm{e}^{\frac{-\left(v-v_{0}\right)^{2}}{w^{2}}}\right]$.

Assuming that this spectrum illuminates the upper atmosphere in which the line presents an absorption profile of width $\Delta v_{D}$ moving at a velocity $v_{z}$ along the vertical direction respect to the lower layers, the anisotropy in the low velocity limit can be written, following Carlin et al. (2012), as

$$
\frac{J_{0}^{2}}{J_{0}^{0}}=\frac{u}{4 \sqrt{2}(2-u)}+a \frac{64-56 u+7 u^{2}}{120(2-u)^{2}\left(1+\alpha^{2}\right)\left(\sqrt{1+\alpha^{2}}-a\right)} \xi^{2},
$$

where $\alpha=\frac{\Delta v_{D}}{w}$ and the velocity gradient $v_{z}$ is written without dimensions as $\xi=\frac{v_{z}}{c} \frac{v_{0}}{w}$. In the absence of both limb darkening and velocity gradients, the anisotropy is 0 . In the absence of a velocity gradient, the anisotropy is due to limb darkening only. More surprisingly, in the absence of limb darkening we can still have an anisotropic radiation field if the upper atmosphere is moving with respect to the static background, and this anisotropy is independent of whether the movement is upward or downward. Of more immediate impact in our present discussion, the sign of the anisotropy modification by velocity gradients is determined by the sign of $a$. That is, for an absorption line $(a>0)$, velocity gradients amplify any anisotropy due to limb darkening, while for an emission line $(a<0)$ the effect is the opposite.
Among the different scenarios sketched above to produce net linear polarisation over the stellar disc of $\chi \mathrm{Cyg}$, we can ascertain the impact of velocity gradient amplification by comparing emission and absorption lines in the linearly polarised spectrum. If in a certain region of the expanding stellar atmosphere there were a gradient of velocity higher than elsewhere, it would amplify the anisotropy of absorption lines that would increase its polarisation and dominate the net polarisation over the disc. But this higher gradient would diminish the anisotropy of emission lines, which would be less polarised than elsewhere over the disc. The net polarisation in emission lines would be dominated by the regions in which local polarisation is the opposite of the region with higher gradients. Summing up, emission and absorption lines, after integration over the disc, present opposite polarisations.

Figure 4 shows the interesting case of the triplet of Ca II lines in the near-infrared. The three components show emission in intensity and, a low red-shifted absorption, most visible at $866.2 \mathrm{~nm}$ and difficult to ascertain in intensity in the two other lines. Such profiles are very common in Mira stars (e.g. Gillet 1988) and are interpreted in the framework of shockwave propagation. The emission component, blue-shifted, originates at the shock front zone, propagating outward, while the red component, in absorption, comes from the unperturbed medium above the shock front. Recalling the pulsation phase of this observation ( $\varphi \sim 0.94$ ), the spectrum has been obtained very close to a maximum light, i.e. during the maximum outward acceleration of the shock. Following our argument comparing emission and absorption lines, in agreement with Eq. (6), and given the observed polarisation signs of the other lines in absorption, the emission component of these lines is expected to show a negative $Q$ signal; the component in absorption would present a positive $Q$ signal, identical to the Na $D$, Ti I, or molecular lines explored above.

This can be verified in Fig. 4. The signals are clear in the Ca II $866.2 \mathrm{~nm}$ and Ca II $854.2 \mathrm{~nm}$ but absent in Ca II $849.8 \mathrm{~nm}$. This is not unexpected in view of the particularities of the intrinsic polarisation of these lines with a strong impact of lower level atomic polarisation in their statistical equilibrium and emission terms (Trujillo Bueno 2003; Carlin et al. 2012; Carlin \& Asensio Ramos 2015).

The effect can be cautiously confirmed by considering the $\mathrm{H} \alpha$ and $\mathrm{H} \beta$ lines (Fig. 5). Interpreting the polarisation of hydrogen lines (of the Balmer series or any other) is extremely complex because of the sensitivity of this atom to all possible polarising effects, and the large regions and conditions on which these lines can form (López Ariste et al. 2005; Casini \& Manso Sainz 2006b,a; Derouich 2007; Štěpán \& Sahal-Bréchot 2008), especially out of local thermodynamical equilibrium (NLTE). But once our working hypotheses are accepted, that is that most lines show intrinsic polarisation and that the net polarisation is apparently due to anisotropies in the radial velocity of the pulsating atmosphere (amplifying the radiation anisotropy), and knowing that with these hypotheses we are able to predict the signs on the combined emission/absorption profiles of the Ca II lines, then it is acceptable to peek into the $\mathrm{H}$ lines for a further confirmation. In the available data, $\mathrm{H} \alpha$ does not show clear signals in intensity, perhaps because the molecular bands strongly absorb the flux originating in the corresponding line forming region. Conversely, the emission profile is clear in the intensity profiles of $\mathrm{H} \beta$ and $\mathrm{H} \gamma$, where the molecular absorption is strongly reduced. The polarisation signals, on the other hand, are strong and unambiguous for the three Balmer lines. And, in broad agreement with the signs observed in the emission part of the Ca II lines (Fig. 4), the emission presents a negative polarisation signal in $Q$, although we also see, particularly 

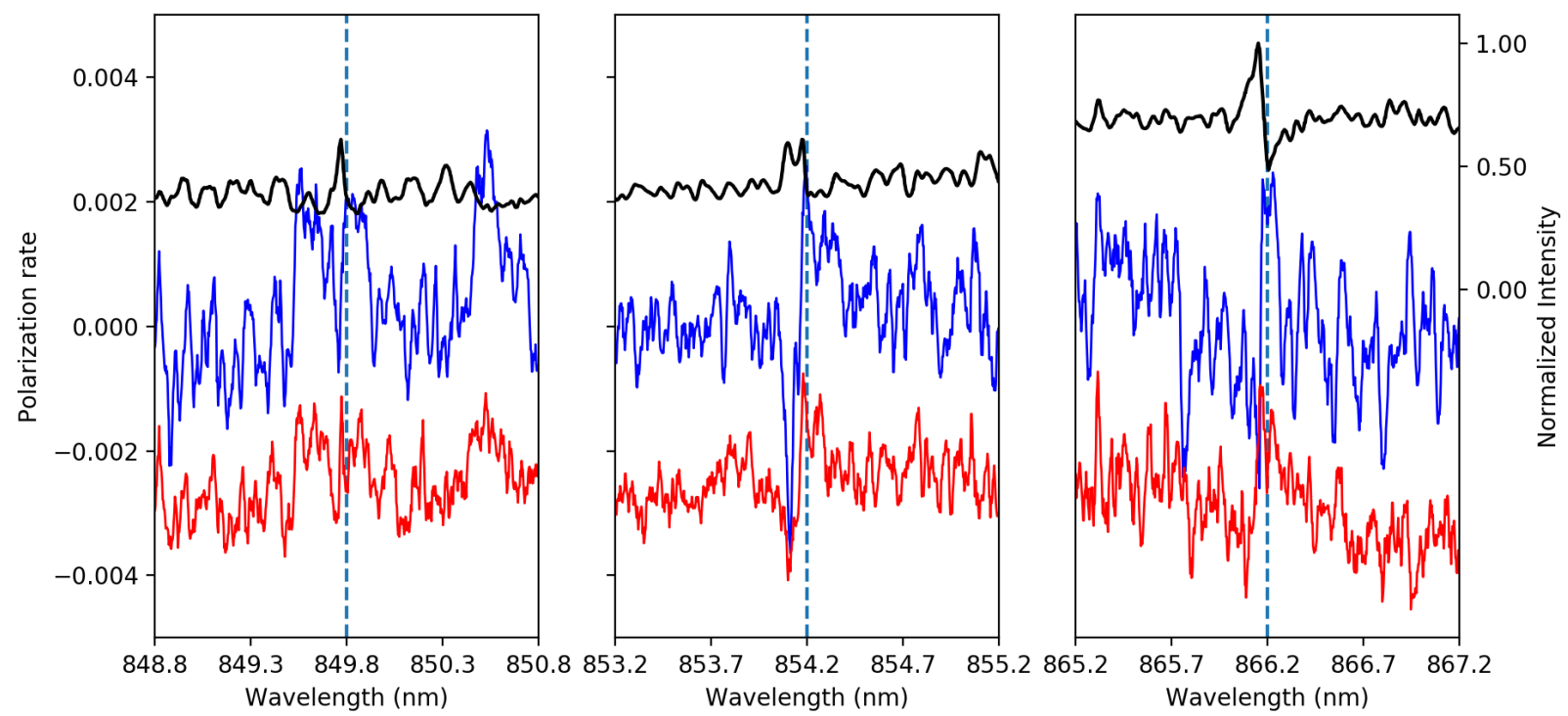

Fig. 4. Spectra of $\chi$ Cyg on September 4, 2007 around the three lines of Ca II triplet in the near-infrared. As in Fig. 2, the black thin line represents intensity (ordinates at right) and Stokes $Q$ and $U$ are shown in red and blue, respectively. The quantity $Q$ has been shifted 0.01 units for clarity. The lines are split into a blue-shifted emission and a red-shifted absorption. The polarisation changes sign accordingly as if dominated by the amplification of anisotropy in the presence of velocity gradients.
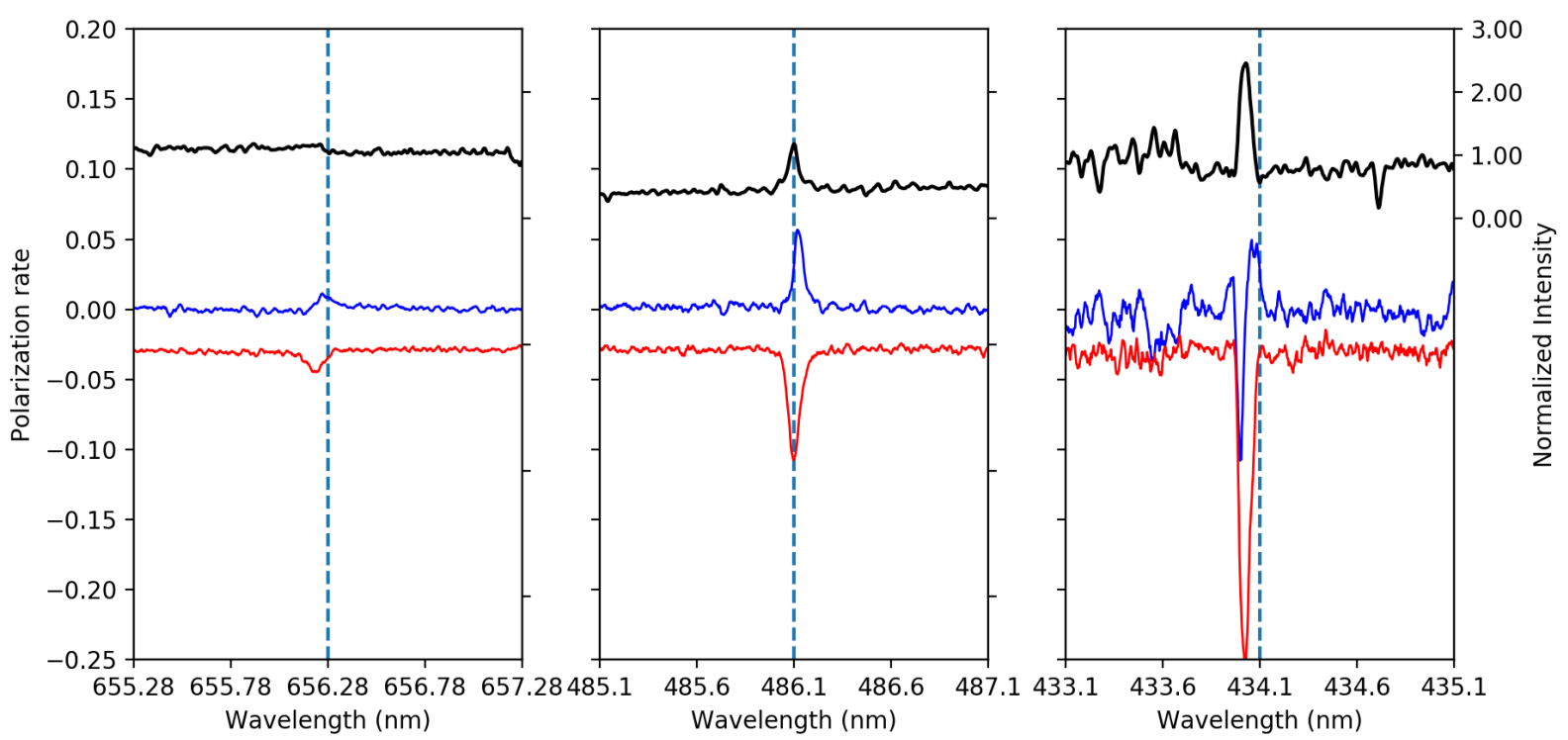

Fig. 5. Spectra of $\chi$ Cyg on September 4, 2007 around $H \alpha$ (left), H $\beta$ (centre) and $H \gamma$ (right). As in Fig. 2, the black thin line represents intensity (ordinates at right) and Stokes $Q$ and $U$ are shown in red and blue, respectively; $Q$ has been shifted 0.01 units for clarity.

in $U$, a red-shifted positive signal that could signal a red-shifted absorption profile that is not visible in the intensity profiles. The complexity of formation of these lines prevents us from interpreting these signal beyond these simple facts and comparisons for these lines.

We thus have a scenario that explains the main features of the linearly polarised spectrum of $\chi$ Cyg. The particular conditions of the atmosphere of this star around its maximum brightness are such that most lines show intrinsic line polarisation. This explains the signal in $\mathrm{Na}$ I $D_{2}$ and its absence in $D_{1}$. The same signal can be expected in all lines, atomic or molecular, with an amplitude scaled by the quantum coefficient $\left(w_{J_{u} J_{l}}^{(2)}\right)^{2}$. We illustrated the case with a Ti I line and with three molecular band heads. The exercise could be continued with other lines, both atomic and molecular, which present the same trends of signs and relative amplitudes in $Q$ and $U$ signals. Following this scenario, the pulsating atmosphere during a maximum light does not have the same radial velocity all over the star. Particular locations are expanding faster than others. Since velocity gradients can amplify the radiation anisotropy of the expanding layers, the light from these locations is more polarised than elsewhere. Consequently, a net linear polarisation appears after integration over the stellar disc. But this amplification of the anisotropy and the polarisation by these velocity gradients only happens for absorption lines. Emission lines recover a smaller anisotropy in the same conditions. For emission lines, the polarisation is reduced by these velocity gradients and the net polarisation is dominated by the opposite signal coming from elsewhere in the star. What we expect is a change of the polarisation sign for emission lines. This is what is observed in two of the lines of the Ca II triplet 
for which there is a $I$ blue-shifted emission and a red-shifted absorption, corresponding to a change of sign in the polarisation as we move from one side to the other of the line profile. This also explains why the Balmer lines, which are mostly in emission, show different and predominantly opposite polarisation signs to the other atomic and molecular lines in absorption. This change in the sign of polarisation in emission and absorption lines cannot be easily explained in the two other scenarios for symmetry breaking. If velocity gradients were identical all over the star, the local polarisation of absorption lines would be larger than those of emission lines everywhere. In that case, if one particular region was either brighter or higher than other regions, the total amount of photons, or the polarisation rate, would be larger than elsewhere. This would be the case for both emission and absorption lines. But the polarisation of emission lines in that brighter region would still be larger than the polarisation of emission lines elsewhere, so that the net polarisation of emission lines would be the one of that brighter region. The net polarisation of both emission and absorption lines would show the sign corresponding to that brighter region, and both emission and absorption lines would show the same sign even though the polarisation amplitude in emission lines would be smaller. This would not explain the change of sign and we would not see see this smaller amplitude of polarisation in emission lines.

\section{Time series of LSD profiles}

Our scenario of anisotropic velocity gradients implies that all absorption lines present the same sign of polarisation. Excluding deep lines for which second order effects such as lower level polarisation and hyperfine structure may be important, the spectral shape of that polarisation signature is the same. As already claimed above, this justifies the use of LSD techniques applied to absorption lines and weighted with the coefficient $\left(w_{J_{u} J_{l}}^{(2)}\right)^{2}$.

The observations of September 4, 2007 are remarkable in that they show clear signatures in individual lines. For this date we compared the LSD profile (Fig. 6) to the signals in individual lines. We recovered the expected signature, proving that LSD really extracts the same signals that we interpreted. The LSD profile also shows a high S/N, its fundamental purpose, and this demonstrates that continuum depolarisation, even if present in some lines, must really be a minor effect: the LSD signal is dominated by intrinsic polarisation. From the point of view of the second spectrum we find that Betelgeuse (dominated by depolarisation) and $\chi$ Cyg (dominated by intrinsic line polarisation) place themselves at the two extremes afforded by Eq. (1), while the Sun is to be found somewhere in between with most of the lines depolarising the continuum and a few remarkable lines showing intrinsic line polarisation. These differences must come from the actual atmospheric structure and the formation regions of the continuum and spectral lines.

Signals are often much lower at other observation dates. We are therefore interested in using the right mask of lines for LSD in the sense that the $\mathrm{S} / \mathrm{N}$ is still high enough, but pollution from molecular lines is as low as possible, given the lack of sufficient information to include molecular lines in the masks. Educated guesses have led us to try to add all atomic lines deeper than $1 \%$ (left plot of Fig. 6), all atomic lines deeper than $40 \%$ (central plot) and (right plot) those lines deeper than $40 \%$ that belong to the following series of atomic species: Ti I, Ti II, Sr I, Sr II, Fe I, Fe II, Cr I, Cr II, Co I, Co II, V I, V II, Ni I, Ni II, K I, K II, Zr I, and Zr II. This last LSD mask, which has over 19000 lines and results in the most clear and unpolluted signal from molecular

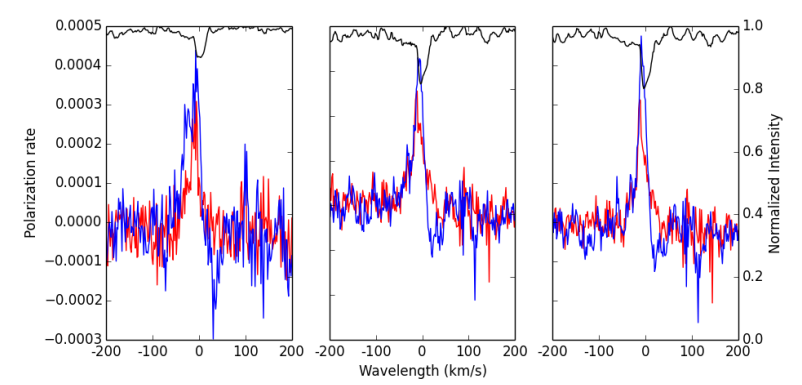

Fig. 6. LSD Spectra of $\chi$ Cyg on September 4, 2007, with lines weighted by $\left(w_{J_{u} J_{l}}^{(2)}\right)^{2} . Q$ is in red, $U$ in blue. Three different masks are used from left to right: all lines deeper than $1 \%$, all lines deeper than $40 \%$, and a selection of lines deeper than $40 \%$ (see text).

lines without loosing much in $\mathrm{S} / \mathrm{N}$, is retained and applied in the following.

Figure 7 shows the LSD profiles for all the available dates. Signals are not always visible, as for example on June 28 and July 19, 2015. We first note that the LSD signals change from cycle to cycle but they are coherent inside each cycle and an evolution in time can be ascertained. We also see that, in general, the polarisation signal appears centred or blue-shifted compared to the line profile minimum. However, for the observation of December 20, 2016, two signals with different signs appear in apparent relation with the double line in the intensity profile. On the other hand, the peaks in $Q$ and $U$ appear at the same wavelength, up to the precision allowed by the S/N. These observed wavelength dependences of the signal are to be understood in the basic scenario which also explains the doubling of the intensity profile that is clearly visible in our data. A brief description was given above when explaining the emission profile of the $\mathrm{Ca}$ II triplet. The observed line doubling in intensity is explained in the framework of the pulsation shock wave: the shockwave physically separates the line forming regions into two parts with two different velocities. The atmosphere above the shock follows a ballistic motion (induced by a former shock propagation), and produces the red-shifted feature, while the region just crossed by the shock is rising, leading to the blue-shifted component. This simple description of a 1D Schwarzschild mechanism (Schwarzschild 1952) needs to be understood in the context of the integration over the stellar disc. Clearly, the two velocities result in a maximum Doppler shift visible as a double line only around disc centre. As we approach the stellar limb the projection onto the line of sight of the velocities drops to zero, cancelling any Doppler shift and eliminating the double line. If a double line is to be seen after integration over the stellar disc, the simplistic 1D picture of the Schwarzschild mechanism must be completed. To address this issue, we followed Bertout \& Magnan (1987) and Wagenblast et al. (1983) and we redrew their scenario for the formation of spectral lines in a moving spherical shell. This is explicitly done in the appendix. The bottom line of their reasoning and our reasoning is to assume that the bulk expansion velocity is higher than the intrinsic width of the line. But, from the point of view of the observer, this is only true for a region around the disc centre, while near the limb the projected velocity is lower than the line width. As shown in the appendix and by Bertout \& Magnan (1987) and Wagenblast et al. (1983), the integral over the stellar disc of the emergent intensity profiles carries a geometrical factor that, under these stated relations between bulk velocity and line width, translates into a wavelength modulation. Independent of any other radiative transfer effect, this disc integration produces 

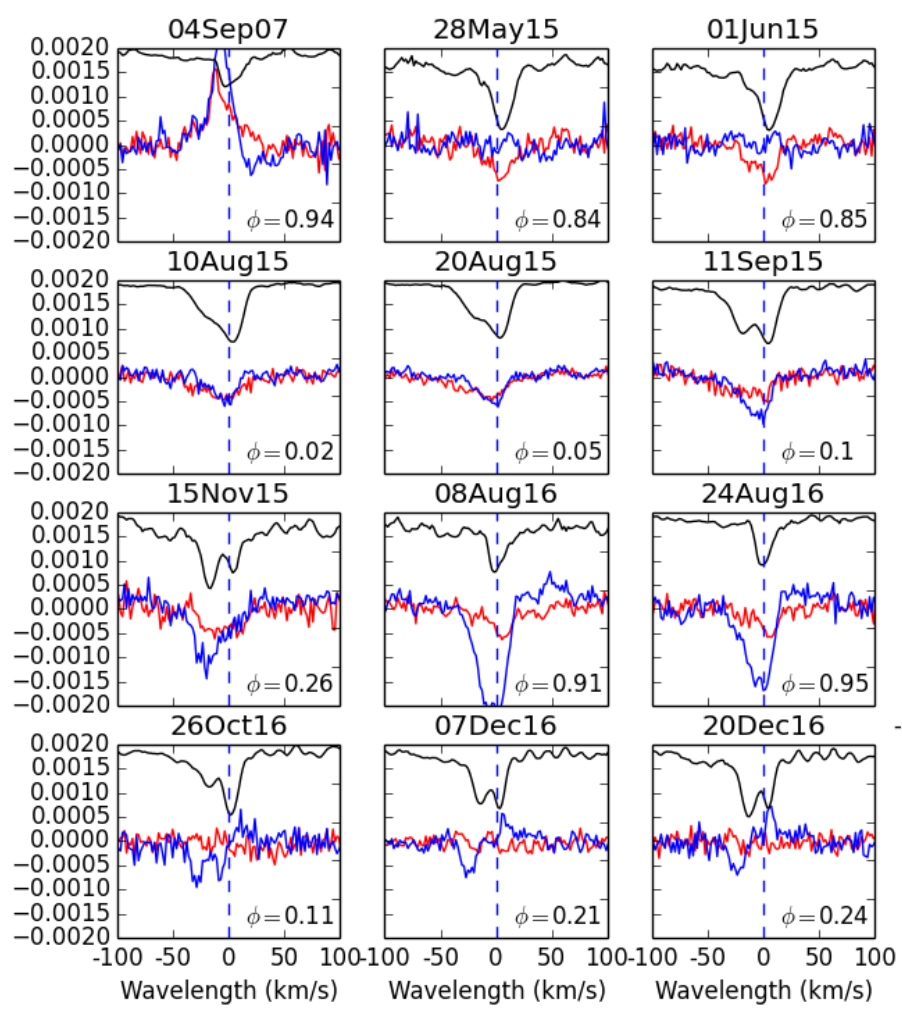

$11 \mathrm{Sep} 15$
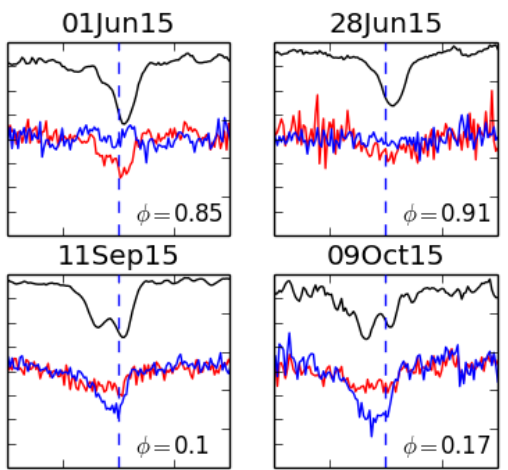

090ct15
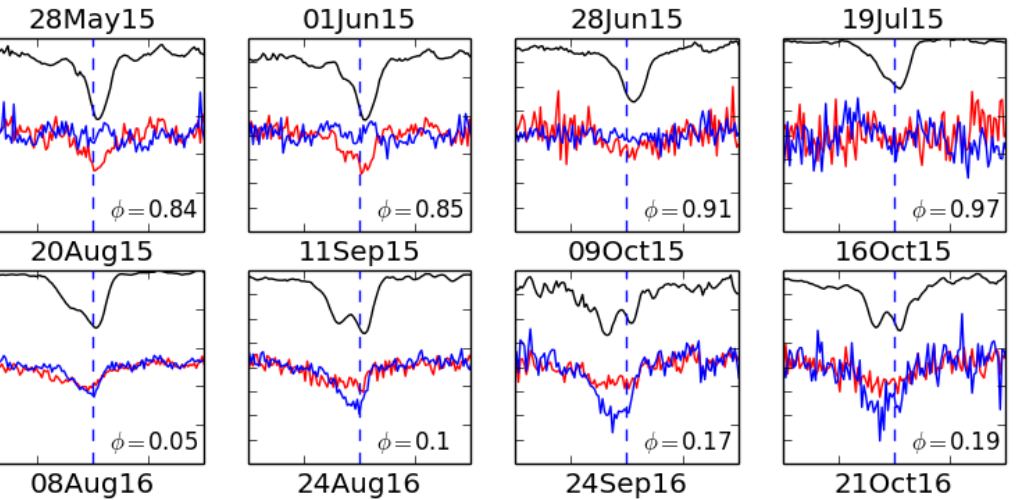

24Aug16

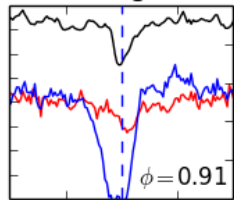

$07 \mathrm{Dec} 16$

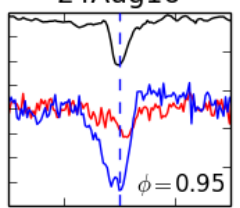

20Dec16
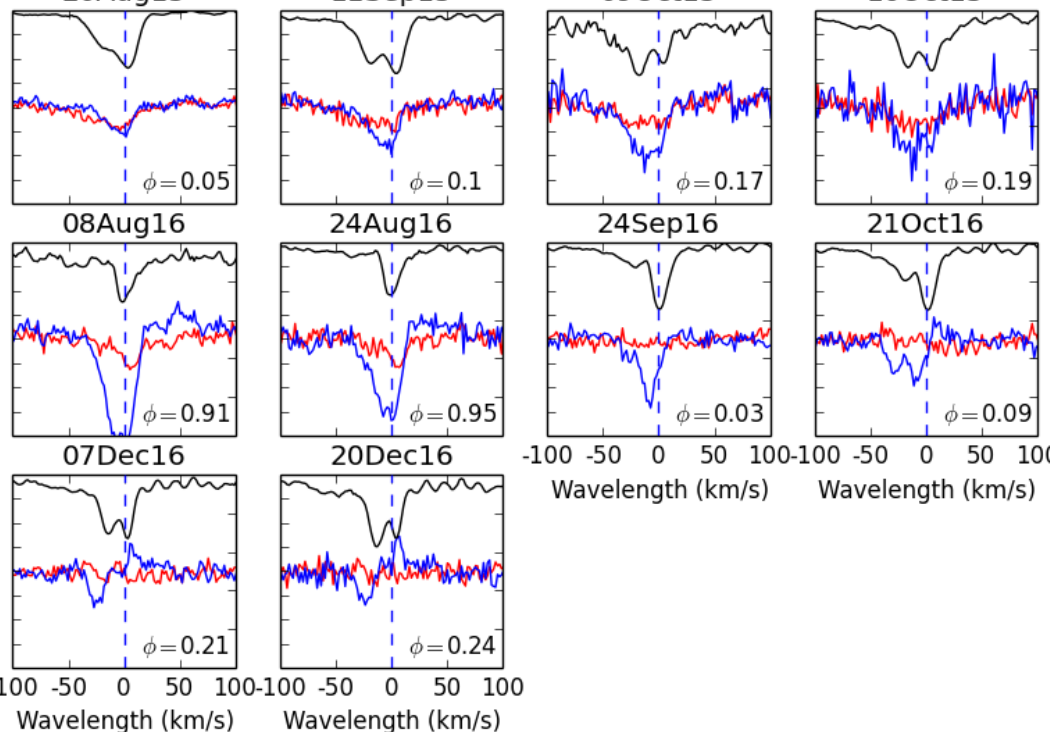

210 ct16

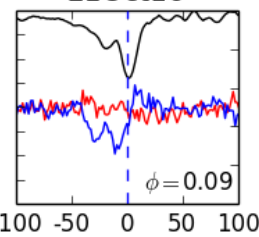

Wavelength $(\mathrm{km} / \mathrm{s}) \quad$ Wavelength $(\mathrm{km} / \mathrm{s})$

Fig. 7. Spectra from LSD of $\chi$ Cyg for each one of the dates for which Stokes $Q$ and $U$ data are available. The vertical dashed line in each plot indicates the heliocentric zero wavelength. The inset text contains the phase at each observation.

doubled profiles, shifted profiles, asymmetric profiles, or flatbottom profiles for the intensity depending on the actual value of the expansion velocity of the shell and in the presence or absence of a contracting shell on top of the expanding shell.

In this very same scenario, polarisation can be computed easily by introducing the factor $\sin ^{2} \theta=1-\mu^{2}$ into the integration over the stellar disc. As shown in the appendix, this dependence must be added to the integrals in a similar way to the geometric factor described by Bertout \& Magnan (1987). This modifies the result in that polarisation comes preferentially from somewhere midway between the centre and the limb. At this position, the Doppler separation is not so large and we expect polarisation to be roughly centred in wavelength when compared with the doubled intensity profiles. On top of this median signal we have to consider the actual details of the position of the anisotropy in velocities, which favours the polarisation signals of certain places of the disc.

The interesting point in the previous description of the line formation in $\chi \mathrm{Cyg}$ is that wavelength can be soundly related to a distance to the disc centre while, as usual, the ratio of $Q$ to $U$ provides information about polar angle. This conclusion leads us, as it did in the work by Aurière et al. (2016) and López Ariste et al. (2018), to the possibility of mapping the velocity field modulation of the pulsation over the disc of $\chi \mathrm{Cyg}$.

Even if its origin is not really known (see next section), the anisotropy of the radial velocity field is required to explain the net observed linear polarisation. In the scenario developed above, this velocity field modulation appears at all observed phases, which are mainly centred on light maxima that correspond to the outward propagation of the shock wave. In the Schwarzschild framework, this is well established by the gradual development of a blue-shifted component that generates line doubling or asymmetric profiles in the $I$ spectra.
However, once the shock wave has crossed the atmosphere, the accelerated matter follows a classical ballistic motion, reaching, around light minimum, its maximum extension. It would be interesting to measure the linear polarisation at this phase, since we do not expect strong inhomogeneities within the velocity field in the line forming region at this phase of maximum extension. Trials to do so have unfortunately been hindered by the even more ubiquitous molecular bands that prevent the computation of any meaningful LSD profile exclusively using masks of atomic lines.

\section{Asymmetries in the shock wave}

The main conclusion of this work has by now been reached. The shock wave in $\chi$ Cyg should present asymmetries in the form of an inhomogeneous radial velocity field. In order to quantify those asymmetries, we can attempt to map them onto the stellar disc using the conclusions from the analysis presented in the previous section on the formation of the polarised line. This cannot be done, however, without assuming a long list of hypotheses and approximations that we try now to make explicit. Seen together, all those hypotheses and approximations imply that the produced maps cannot be seen at this point as the actual representation, point by point, of the actual velocity field in the pulsation wave of $\chi$ Cyg. But these maps can be a source of information on spatial and temporal scales of the inhomogeneities and their relative importance with respect to the average velocity of the shock.

With this word of caution, we start by recalling that the ratio $Q / U$ provides the polar angle $\chi$ of regions over the disc that contribute with a larger polarisation because of a higher velocity field. But they do so through the expression

$\tan 2 \chi=\frac{U}{Q}$, 
so that a $180^{\circ}$ ambiguity pops up: the emitting region of the polarisation excess may be at one side or the other of a given diameter across the stellar disc. In our maps we arbitrarily selected just one of the two possibilities.

To explain the observed linear polarisation profiles, in our model we assumed that the only source of linear polarisation is scattering and that no phenomena other than velocity gradients modify this polarisation. But it is well known that magnetic fields, through the Hanle effect, can both diminish this scattering polarisation and rotate the polarisation plane (e.g., Landi Degl'Innocenti \& Landolfi 2004). Less known is that nonvertical velocity fields can produce the same effect as the Hanle effect: that is, it can depolarise and rotate the polarisation plane (de Kertanguy 1998; Landi Degl'Innocenti \& Landolfi 2004). This rotation of the local polarisation plane can mask the dependence of the $Q / U$ ratio on the polar angle. Thus we are making the hypothesis in this work that the velocity field is strictly radial and that no magnetic fields are present; this last approximation made in spite of the measurement of such fields through the observation of the Zeeman effect in the circular polarisation of the lines (Fabas et al. 2011; Lèbre et al. 2014). While imposing a radial velocity field in a scenario of a radially pulsating star may appear as a natural hypothesis, overlooking the impact of actually measured magnetic fields may be judged inappropriate. It can be argued however that the Zeeman effect is sensitive to fields much larger than the Hanle effect, so that those fields measured through the Zeeman effect are most probably relatively strong fields (above $100 \mathrm{G}$ ), which do not influence scattering linear polarisation. If the measured circular polarisation amplitudes are small $(1-2 \mathrm{G})$ it is just because of polarity mixing or dilution in a non-magnetic atmosphere and not because of intrinsically weak fields.

We also assume that at any time the star is spherical. If it were an ellipsoid, as in the case of other stars with huge rotational speeds, there would be a natural source of net polarisation due to this shape. $\chi$ Cyg is not a rapid rotator and if nevertheless this was the origin of the observed net polarisation, we would have failed to interpret the different signs of polarisation in absorption and emission lines, which we have been able to accomplish. So this must be at most a second order contribution.

Another approximation comes when inferring the distance $\mu$ to the disc centre. Our approach has been that of the simplified model used for the imaging of Betelgeuse made by Aurière et al. (2016). Each wavelength in the profile corresponds to a unique point on the stellar disc of $\chi$ Cyg. Thus, the recovered information concerns just as many points as there are in the spectral binning of our profiles, i.e. about 30 . This is obviously a very rough approximation. In the case of Betelgeuse, it has been demonstrated (López Ariste et al. 2018) that such simple modelling can provide, at best, only basic information of the brightest points (or the higher velocity points in our case). But this approximation can also be the source of strange unphysical distributions. For example Fig. 8, in which the velocity is represented as arrows over the visible hemisphere of the star, shows strange filaments of high velocities over the disc that look similar in shape to the examples shown by López Ariste et al. (2018) and have their origin in the drastic identification of one wavelength, one point. The right approach would have been to propose a model for the velocity distribution over the stellar disc in terms of spherical harmonics or others. However at this point we miss the broader view of what such a distribution may look like so we preferred the basic approximation of one wavelength, one point from which at least we can retrieve some information on the highest velocity inhomogeneities present.

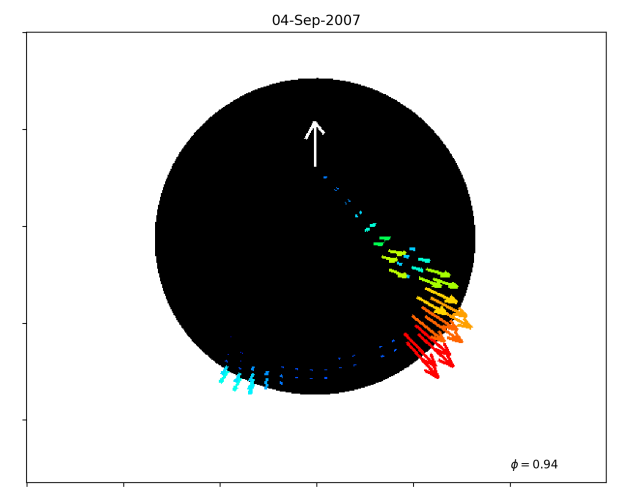

Fig. 8. Map of the velocity field on $\chi$ Cyg on September 4, 2007. A uniform radial velocity is assumed except for those places at which polarisation indicates higher velocities. The arrow length and the colour code indicate redundantly the magnitude of the velocity in terms of the background velocity field for those places at which this velocity is different. The white arrow represents the line of sight.

With these caveats in mind, Fig. 9 shows the inferred maps of the pulsation velocity through the sequence of observation dates with the same representation as Fig. 8. To build each of these we went over the $Q$ and $U$ profiles of each date and assigned a distance to disc centre $\mu$ to every wavelength assuming a constant velocity projected onto the line of sight over the whole disc. This is simplistic and oversees our conclusion about velocity gradients but, once again, our goal is to get a first picture of the spatial and temporal scales of those gradients. We next assigned an azimuthal angle $\chi$ determined from the ratio of $Q$ to $U$ amplitudes at that wavelength. At that point $(\mu, \chi)$ over the stellar disc we drew an arrow proportional to the amplitude of polarisation $\sqrt{\left(Q^{2}+U^{2}\right)}$ and representing a qualitative measurement of the gradients of the velocity at that point. Since the $3 \mathrm{D}$ projection may make it difficult to ascertain the actual length of every arrow, we also coded this information in colour. As expected from the coherency of the signals, the direction of higher velocities is approximately constant around each maximum. The maxima of 2015 and 2016 also appear to share a common direction, but this direction is orthogonal to that seen in the maximum of 2007. This would exclude a permanent preference of direction related, for example, to the rotation axis of the star. There is also no particular correlation between the date of the maximum brightness and the date of maximum polarisation.

\section{Conclusions}

The observation of net linear polarisation in the spectral lines of $\chi$ Cyg around three different pulsation maxima has led us to conclude that the velocity of the pulsation, while still radial, is not homogeneous in velocity over the stellar disc. The source of these inhomogeneities is not known but since they change in shape and position from one maximum to the next it must be concluded that the source is not a permanent feature of the star. It is nevertheless a recurrent feature, cycle after cycle, so we must also conclude that it is somehow related to the shock associated with the pulsation.

We can speculate on the interaction between the convective motions and this pulsation shock as a possible culprit: strong localised convective up-flows would add up velocities with the shock, resulting in localised higher velocities of pulsation. Another hypothetical source of asymmetries in the velocities would be the interaction of the shock with returning material 


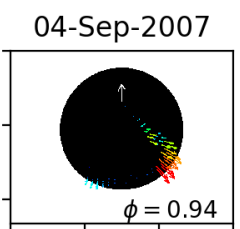

10-Aug-2015

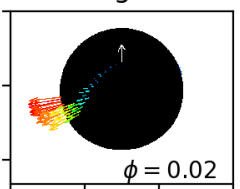

15-Nov-2015

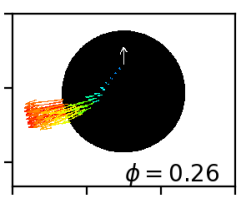

26-Oct-2016

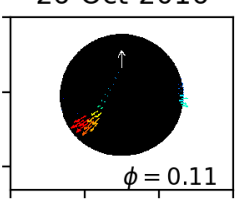

28-May-2015

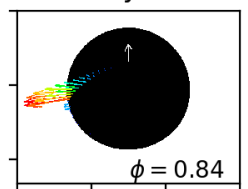

20-Aug-2015

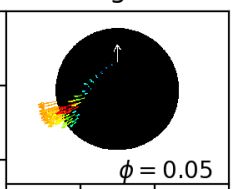

08-Aug-2016

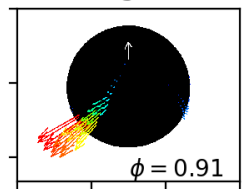

07-Dec-2016

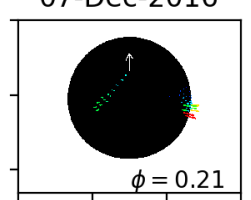

01-Jun-2015

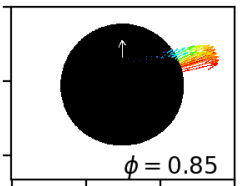

11-Sep-2015

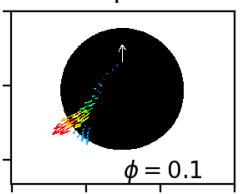

24-Aug-2016

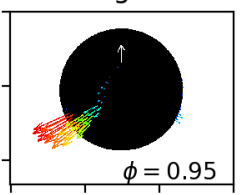

20-Dec-2016
28-Jun-2015

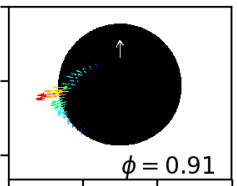

09-Oct-2015

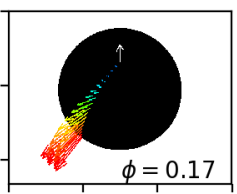

24-Sep-2016
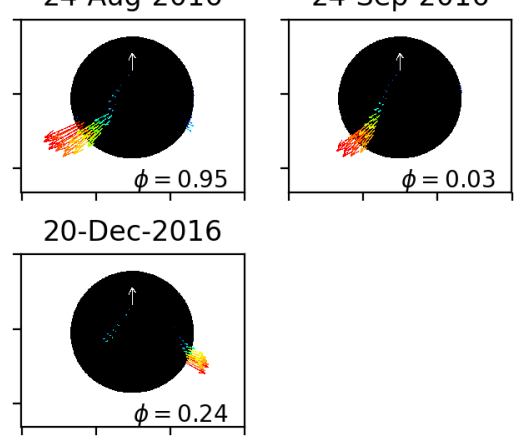

19-Jul-2015

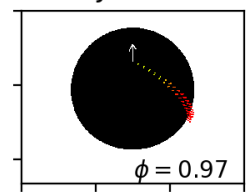

16-Oct-2015

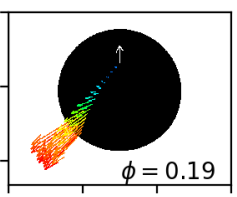

21-Oct-2016

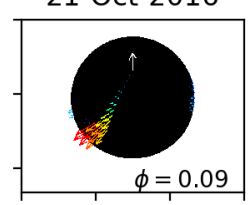

Fig. 9. Maps of the velocity field on $\chi$ Cyg. A uniform radial velocity is assumed except for those places in which polarisation indicates higher velocities. The arrow length and the colour code indicate redundantly the magnitude of the velocity in terms of the background velocity field for those places at which this velocity is different. The white arrow represents the line of sight and the inset text gives the pulsation phase.

from a previous pulsation cycle. These are the questions that future work will have to explore.

Inferring that an inhomogeneous velocity field is the cause of the observed linear polarisation signal has required the interpretation of this signal as due to the intrinsic polarisation of each individual spectral line. We disentangled intrinsic polarisation from depolarisation of the continuum through the inspection of individual lines; the two terms appear in the equation for the transfer of linear polarisation in the presence of scattering. But we also demonstrated that line addition, through techniques such as LSD, can help to disentangle the two processes as well by comparing the net signal recovered when different weights for each individual line are used. Line depolarisation signatures are enhanced when the line depression is used as weight, while intrinsic polarisation is brought up by using the quantum $w_{J_{u} J_{l}}^{(2)}$ coefficient of the line. This protocol allows us to reach a conclusion on the origin of observed linear polarisation, even in the case in which the polarisation signal on individual lines is too weak.

Acknowledgements. This work was supported by the "Programme National de Physique Stellaire" (PNPS) of CNRS/INSU co-funded by CEA and CNES.

\section{References}

Alvarez, R., Jorissen, A., Plez, B., Gillet, D., \& Fokin, A. 2000, A\&A, 362, 655

Alvarez, R., Jorissen, A., Plez, B., et al. 2001, A\&A, 379, 305

Asensio Ramos, A., \& Trujillo Bueno, J. 2005, ApJ, 635, L109

Asensio Ramos, A., \& Trujillo Bueno, J. 2006, ApJ, 636, 548

Aurière, M., López Ariste, A., Mathias, P., et al. 2016, A\&A, 591, A119

Bertout, C., \& Magnan, C. 1987, A\&A, 183, 319

Boyle, R. P., Aspin, C., Coyne, G. V., \& McLean, I. S. 1986, A\&A, 164, 310

Carlin, E. S., \& Asensio Ramos, A. 2015, ApJ, 801, 16

Carlin, E. S., Manso Sainz, R., Asensio Ramos, A., \& Trujillo Bueno, J. 2012, ApJ, 751, 5
Carlin, E. S., Asensio Ramos, A., \& Trujillo Bueno, J. 2013, ApJ, 764, 40 Casini, R., \& Manso Sainz, R. 2006a, Solar Polarization 4 (San Francisco: ASP), 358,429

Casini, R., \& Manso Sainz, R. 2006b, J. Phys. B At. Mol. Phys., 39, 3241

Casini, R., Landi Degl'Innocenti, E., Landolfi, M., \& Trujillo Bueno, J. 2002, ApJ, 573, 864

Clarke, D., \& Schwarz, H. E. 1984, A\&A, 132, 375

de Kertanguy, A. 1998, A\&A, 333, 1130

Derouich, M. 2007, A\&A, 466, 683

Doherty, L. R. 1986, ApJ, 307, 261

Donati, J.-F., Semel, M., Carter, B. D., Rees, D. E., \& Collier Cameron, A. 1997, MNRAS, 291, 658

Duthu, A., Herpin, F., Wiesemeyer, H., et al. 2017, A\&A, 604, A12

Fabas, N., Lèbre, A., \& Gillet, D. 2011, A\&A, 535, A12

Fadeyev, Y. A., \& Gillet, D. 2004, A\&A, 420, 423

Fokin, A. B. 1991, MNRAS, 250, 258

Gillet, D. 1988, A\&A, 192, 206

Gillet, D., Maurice, E., \& Baade, D. 1983, A\&A, 128, 384

Gillet, D., Ferlet, R., Maurice, E., \& Bouchet, P. 1985, A\&A, 150, 89

Herpin, F., Baudry, A., Thum, C., Morris, D., \& Wiesemeyer, H. 2006, A\&A, 450,667

Kochukhov, O., Makaganiuk, V., \& Piskunov, N. 2010, A\&A, 524, A5

Landi Degl'Innocenti, E., \& Landolfi, M. 2004, Polarization in Spectral Lines (Dordrecht: Kluwer Academic Publishers), 307

Lèbre, A., Aurière, M., Fabas, N., et al. 2014, A\&A, 561, A85

Lèbre, A., Aurière, M., Fabas, N., et al. 2015, IAU Symp., 305, 47

Leroy, J. L. 1972, A\&A, 19, 287

López Ariste, A., Casini, R., Paletou, F., et al. 2005, ApJ, 621, L145

López Ariste, A., Mathias, P., Tessore, B., et al. 2018, A\&A, 620, A199

Mathias, P., Aurière, M., López Ariste, A., et al. 2018, A\&A, 615, A116

Paletou, F. 2012, A\&A, 544, A4

Schwarzschild, M. 1952, in Transactions of the IAU, ed. P. Oosterhoff (Cambridge: Cambridge University Press), VIII, 811

Stenflo, J. O. 2005, A\&A, 429, 713

Stenflo, J. O., \& Keller, C. U. 1997, A\&A, 321, 927

Štěpán, J., \& Sahal-Bréchot, S. 2008, Proc. SF2A-2008, 573

Trujillo Bueno, J. 2003, Solar Polarization (San Francisco: ASP), 307, 407

Trujillo Bueno, J., Casini, R., Landolfi, M., \& Landi Degl'Innocenti, E. 2002, ApJ, 566, L53

Vlemmings, W. H. T., Khouri, T., Martí-Vidal, I., et al. 2017, A\&A, 603, A92

Wagenblast, R., Bertout, C., \& Bastian, U. 1983, A\&A, 120, 6 


\section{Appendix A: Integration over the disc of intensity and polarisation line profiles from moving spherical shells}

The doubling of the intensity profiles and, by extension, the polarisation profiles observed in $\chi$ Cyg is attributed to the presence of one or more spherical shells in movement. The Schwarzschild mechanism (Schwarzschild 1952) is then usually recalled (Alvarez et al. 2000) to explain that, at disc centre, each moving layer produces an individual line Doppler shifted by its velocity: if one layer is moving outward and another layer is falling back, two lines are produced. Cartoons of this mechanism are given by Alvarez et al. (2000). None of those cited works however puts any attention to the fact that, as said above, this justifies only the profile that would be observed at disc centre. Assuming that the velocities of the moving shells are radial and equal to $+v$ and $-v$, the two lines are Doppler shifted at disc centre by $2 v$, but at the limb, the null projection over the line of sight produces the addition of the two lines without any frequency shift. By integration over the disc, all cases of line doubling from 0 at the limb to $2 v$ at disc centre appear at different proportions and produce bottom flattened profiles (see as examples the left profiles in Figs. A.2 and A.3 later). Since these are not the observed profiles, we must conclude that the integration over the disc is a critical aspect of the line doubling and it is confounding that so many works ignore it.

Polarisation is produced preferentially at high scattering angles. Thus, it is close to the limb that we expect most of the polarisation to come from. Following the previous scheme, at the limb the projected velocities of the moving shells onto the line of sight are zero, and the largest amount of polarisation must be emitted at an unshifted wavelength. If such a statement were true, we would expect no line doubling in the polarisation profile, but rather a well-centred linear polarisation at the zero rest velocity of the star. This is not what is observed either.

To understand the polarisation line profiles, we need to understand how to integrate correctly over the stellar disc and, as a particular case, how the intensity line profiles result in a doubled profile after disc integration. The best explanation of how this integration is done is found in the work of Bertout \& Magnan (1987) and Wagenblast et al. (1983). Those authors already claim in their respective introductions that their purpose is, indeed, to explain the line doubling observed in Mira stars. There are several reasons why we found it interesting to reproduce the arguments and cases of Bertout \& Magnan (1987) in this appendix. First later literature on the spectra of Mira stars has plainly ignored those works; second, these works play a critical role in explaining how the 1D Schwarzschild mechanism can still be seen as a cartoon explanation of what happens even after disc integration; and third, we needed to reproduce those computations, while introducing a $\sin ^{2} \mu$ factor in the integrals to explain the line doubling in linear polarisation.

The star is assumed to produce a continuum spectrum which is then absorbed by an optically thick spherical layer. Bertout \& Magnan (1987) also explored the cases of optically thin layers, but we go straight into the case of interest to us and illustrate exclusively the optically thick case. The spherical layer is assumed to move with a velocity $v(r)$ which depends on the distance $r$ to the centre of the star (see Fig. A.1 for the graphical definition of $r$ and other parameters of interest). The layer itself has a thickness given by the difference between the inner and outer radii $r_{1}$ and $r_{2}$. We observe the star along a given direction $z$. The transverse distance $\mu$ is defined in the main text as the distance to the centre of the disc in the plane perpendicular

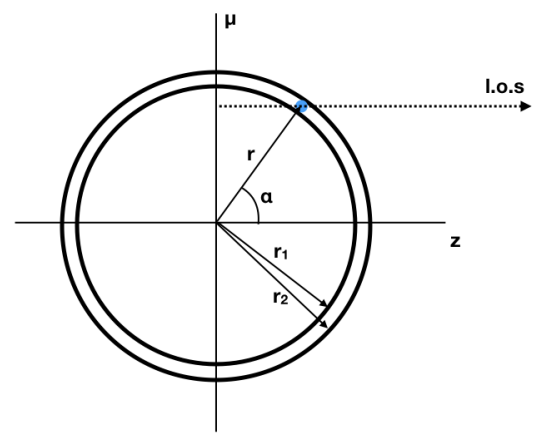

Fig. A.1. Geometry of the expanding spherical shell.

to $z$. A point in a given line of sight at $\mu$ is defined by coordinates $\mu$ and $z$, but also by a radius $r$ and an angle $\alpha$. We have that

$r^{2}=\mu^{2}+z^{2}$

and

$\cos \alpha=\frac{z}{r}=\frac{z}{\sqrt{\mu^{2}+z^{2}}}$.

Along a given line of sight $\mu$, radiative transfer requires the solution of an integral for the specific intensity at a given frequency $v$,

$I_{\nu}(p)=\int S[r] \mathrm{e}^{-\tau_{\nu}(p, z)} \mathrm{d} \tau_{v}$,

where $S$ is the source function and $\tau_{v}$ is the optical opacity at frequency $v$, which we can write as

$d \tau_{v}=-k \phi\left(v-v_{0} \frac{v}{c} \cos \alpha-v_{0}\right) \mathrm{d} z$.

Symbols in this expression have their usual meanings: $k$ is an absorption coefficient and $\phi$ is the line profile centred at $v_{0}$ except for Doppler shifts.

One of the main arguments of Bertout \& Magnan (1987) is that the line doubling is a purely geometrical effect, and this cannot be better demonstrated than by simplifying radiative transfer to its bare fundamentals. Therefore, and because of our assumption of an optically thick layer, we can just impose that, independent of $\mu$, the specific intensity is just an appropriately defined average $\langle S\rangle$ of the source function, Doppler shifted to the frequency $v_{0} \frac{v}{c} \cos \alpha$. Correct radiative transfer calculations, as done by Fokin (1991) introduce an improved line profile shape and correct line depressions. But the basic line doubling in the presence of a moving spherical shell can be retrieved in this bare scenario. With an infinitely thin line profile, for a given $\mu$, the frequency $v$ at which $\langle S\rangle$ is non-zero is given just by

$v-v_{0}-v_{0} \frac{v}{c} \cos \alpha=0$.

We can rewrite this condition in terms of $\mu$ by developing $\cos \alpha$ as follows:

$\mu^{2}=r^{2}\left[1-\left(\frac{c}{v_{0} v}\right)^{2}\left(v-v_{0}\right)^{2}\right]$.

From this, the contribution of a differential element $\mathrm{d} \mu$ over the disc can be obtained by differentiation. Recalling that $v$ is a function of $r$ and making use of the fact that

$\frac{r}{v} \frac{\mathrm{d} v}{\mathrm{~d} r}=\frac{\mathrm{d} \log v}{\mathrm{~d} \log r}=a$, 
we can write this differential contribution as

$\mu \mathrm{d} \mu=2 r \mathrm{~d} r\left[1-(1-a)\left(\frac{c}{v_{0} v}\right)^{2}\left(v-v_{0}\right)^{2}\right]+r^{2}\left(\frac{c}{v_{0} v}\right)^{2}\left(v-v_{0}\right) \mathrm{d} v$.

Our calculations up to this point have transformed the original integration over opacity $\mathrm{d} \tau$ into an integration over the disc which reduces to an integration over the radial coordinate $\mathrm{d} \mu$. Then, because of the relationship between the position over the disc and the Doppler velocity of the expanding or contracting shells, this reduces into the sum of an integration over spectral frequency $\mathrm{d} v$ and a second one through the thickness of the shell $\mathrm{d} r$ with a strong weight favouring profiles emerging from disc centre over those from the limb, as we show below.

Two different cases can be considered in view of these two terms at the right side of this equation: either the velocity of the spherical shell is such that macroscopic Doppler shifts are much larger than the width of the spectral line, and in such cases we can neglect the term in $\mathrm{d} v$; or the line is broader than the Doppler shifts owing to the moving shell and it is the first term in $\mathrm{d} r$ that we can neglect. This second case produces what Bertout \& Magnan (1987) referred to as sawtooth profiles, which they considered as the probable description of the line doubled lines in Mira stars. However, posterior works proved that the shocks of $\chi$ Cyg and other Mira stars present velocities with Doppler shifts much larger than the line thermal width. These works force us to be in the first case above, where we can approximate

$\mu \mathrm{d} \mu=2 r \mathrm{~d} r\left[1-(1-a)\left(\frac{c}{v_{0} v}\right)^{2}\left(v-v_{0}\right)^{2}\right]$.

This expression allows us to easily write and compute the disc integration of the emergent profiles. The observed flux at frequency $v$ is given by the integral

$$
\begin{aligned}
& F_{v}=\int_{0}^{2 \pi} \mathrm{d} \chi \int_{0}^{r} I_{v}(p) p \mathrm{~d} p \\
& =2 \pi\langle S\rangle \int_{0}^{r} r \mathrm{~d} r\left[1-(1-a)\left(\frac{c}{v_{0} v}\right)^{2}\left(v-v_{0}\right)^{2}\right] .
\end{aligned}
$$

Let us finally assume that the shock layer is thin enough so that we can make $v$ constant through the layer. This allows us to write the final and simple expression

$F_{v}=\pi\langle S\rangle\left[1-(1-a)\left(\frac{c}{v_{0} v}\right)^{2}\left(v-v_{0}\right)^{2}\right]\left(r_{2}^{2}-r_{1}^{2}\right)$

The frequency dependence of $F_{v}$ comes exclusively from the equations that describe $\mathrm{d} r$ in terms of $\mathrm{d} \mu$. That is, the observed flux presents a shape in frequency which is determined not by radiative transfer but by a geometric factor alone. Depending on the value of $a$, the logarithmic gradient of the velocity, we find two interesting results. If $a=1$, that is, if the layer does not move or if it moves with a velocity proportional to $r$, then

$F=\pi\langle S\rangle\left(r_{2}^{2}-r_{1}^{2}\right)$

independent of frequency. This is a bottom-flat square profile where no line doubling can be seen. This is the case illustrated in the left plots of Figs. A.2 and A.3, and shows what a straightforward disc integration of the 1D Schwarzschild mechanism would
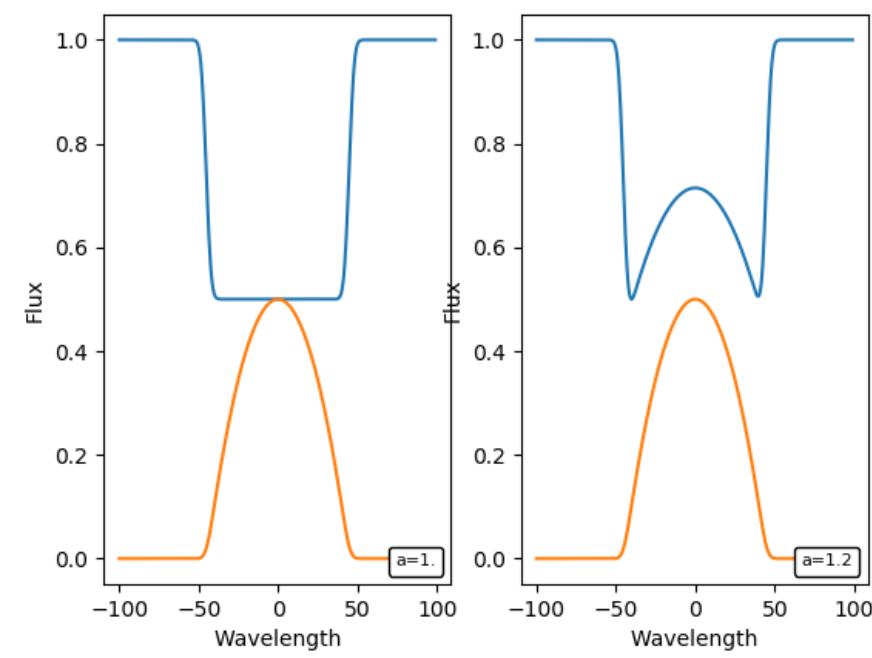

Fig. A.2. Examples of line formation following Bertout \& Magnan (1987). Left: a basic Schwarzschild mechanism produces a flat bottom profile captured by setting $a=1$. Right: two accelerating spherical shells with acceleration $a=1.2$ : one expanding and the second falling, producing the line doubling. The red profiles correspond to the linear polarisation profiles.

produce. On the other hand, if $a>1$ (the layer is expanding in an accelerated motion), then $F_{v}$ has a parabolic shape in which the disc centre contributes to the blue edge of the line with weight one, and points further and further from disc centre contribute at frequencies nearer and nearer to the central frequency $v_{0}$ but with a diminishing factor $\left(v-v_{0}\right)^{2}$ multiplying those contributions. The result is a line which grows towards its blue edge and leaves the line centre with less photons. A second layer, falling into the star from a previous pulsation, produces the same profile red-shifted. This is the line doubling observed in $\chi$ Cyg explained exclusively by a geometric effect. Since the maximum depth of the line is attained at the maximum of $\left(v-v_{0}\right)^{2}$, that is from the contributions from the disc centre where the expansion velocity coincides with the Doppler shift, we recover a scenario in which the 1D Schwarzschild mechanism correctly guesses where the doubled lines are found, even if it misses completely the meaning of those doubled profiles.

In our observations the doubled line is not always apparent, but the intensity profile appears to be displaced from the line centre. It is sufficient to eliminate the falling layer from the computations and assume that only the expanding layer is emitting light. We illustrate this case in Fig. A.3. The line appears both displaced and asymmetric.

These simple calculations confirm one of the most important assumptions in our work, which is that every frequency of the double line corresponds to a Doppler shift coming from the projection of the velocity $v$ onto the line of sight $\frac{v}{c} \cos \mu$. This justifies our assignment of every frequency to one distance to the disc centre $\mu$, opening the path to mapping the velocity inhomogeneities over the disc.

The same computations can be now repeated for the polarisation by just introducing the $\sin ^{2} \mu$ factor into Eq. (A.3) as follows:

$Q_{\nu}(p)=\int S[r] \sin ^{2} \mu \mathrm{e}^{-\tau_{\nu}(p, z)} \mathrm{d} \tau_{v}$.

In the intensity case, geometry alone enhanced light coming from disc centre. We expect that the $\sin ^{2} \mu$ factor takes the geometrical effect of the expanding layer into account by 

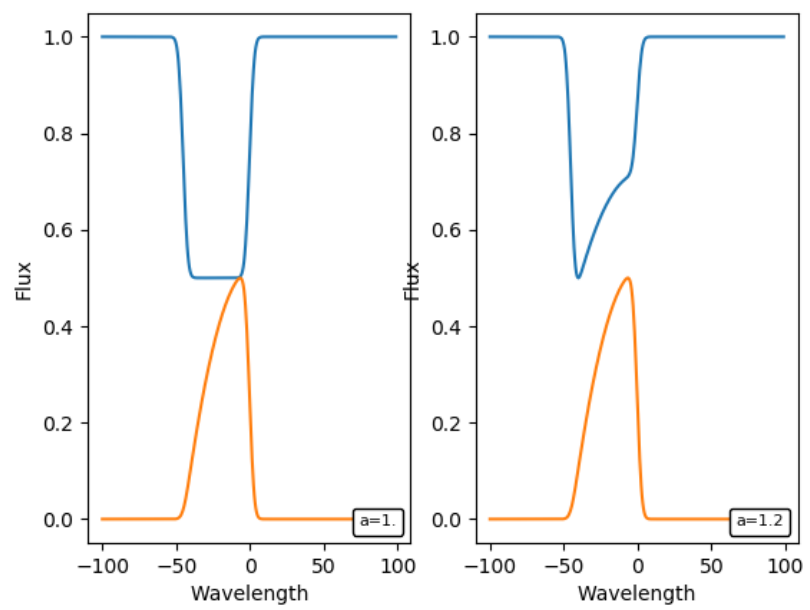

Fig. A.3. As in Fig. A.2, examples of line formation following Bertout \& Magnan (1987). Left: a basic Schwarzschild mechanism produces a flat bottom profile captured by setting $a=1$. Right: two accelerating spherical shells with acceleration $a=1.2$ : one expanding and the second falling, producing line doubling. The maximum contribution comes from the centre of the disc, the limbs being weighed down by the geometry of the problem, so the profile roughly behaves as if coming from 1D radiative transfer at disc centre. The red profiles correspond to the linear polarisation profiles. enhancing light coming from the limb. After rewriting this new factor in terms of the line frequencies and the coordinate $r$ using exactly the same conditions, approximations, and developments of the intensity case, we find that Eq. (A.11) becomes

$$
\begin{aligned}
F_{v}^{Q}= & \pi\langle S\rangle\left[1-\left(\frac{c}{v_{0} v}\right)^{2}\left(v-v_{0}\right)^{2}\right] \\
& \times\left[1-(1-a)\left(\frac{c}{v_{0} v}\right)^{2}\left(v-v_{0}\right)^{2}\right]\left(r_{2}^{2}-r_{1}^{2}\right) .
\end{aligned}
$$

The new factor in that expression is identical except for the absence of the $(1-a)$ factor. The red lines in Figs. A.2 and A.3 show that the expected polarisations in the two cases of accelerated expansion and in the presence or absence of a falling shell. What we see is that the polarisation profile is always better centred than the intensity profile. In the case of the presence of both the expanding and falling shells, the polarisation profile is symmetric and centred, not presenting the double line that the intensity profile shows. When just the expanding shell is present, the maximum polarisation emission is expected very close to the central frequency, unlike the intensity profile which is shifted. In both cases, there is a clear correspondence between wavelength and distance to disc centre. 